\title{
Direct Numerical Simulation of Hypersonic Boundary-Layer Transition over a Blunt Cone
}

\author{
Xinliang $\mathrm{Li}, \stackrel{*}{*}$ Dexun $\mathrm{Fu}$, and Yanwen Ma \\ Institute of Mechanics, Chinese Academy of Sciences, 100080 Beijing, People's Republic of China
}

DOI: $\underline{10.2514 / 1.37305}$

\begin{abstract}
Direct numerical simulation of transition flow over a blunt cone with a freestream Mach number of 6, Reynolds number of 10,000 based on the nose radius, and a 1-deg angle of attack is performed by using a seventh-order weighted essentially nonoscillatory scheme for the convection terms of the Navier-Stokes equations, together with an eighth-order central finite difference scheme for the viscous terms. The wall blow-and-suction perturbations, including random perturbation and multifrequency perturbation, are used to trigger the transition. The maximum amplitude of the wall-normal velocity disturbance is set to $1 \%$ of the freestream velocity. The obtained transition locations on the cone surface agree well with each other for both cases. Transition onset is located at about 500 times the nose radius in the leeward section and 750 times the nose radius in the windward section. The frequency spectrum of velocity and pressure fluctuations at different streamwise locations are analyzed and compared with the linear stability theory. The second-mode disturbance wave is deemed to be the dominating disturbance because the growth rate of the second mode is much higher than the first mode. The reason why transition in the leeward section occurs earlier than that in the windward section is analyzed. It is not because of higher local growth rate of disturbance waves in the leeward section, but because the growth start location of the dominating second-mode wave in the leeward section is much earlier than that in the windward section.
\end{abstract}

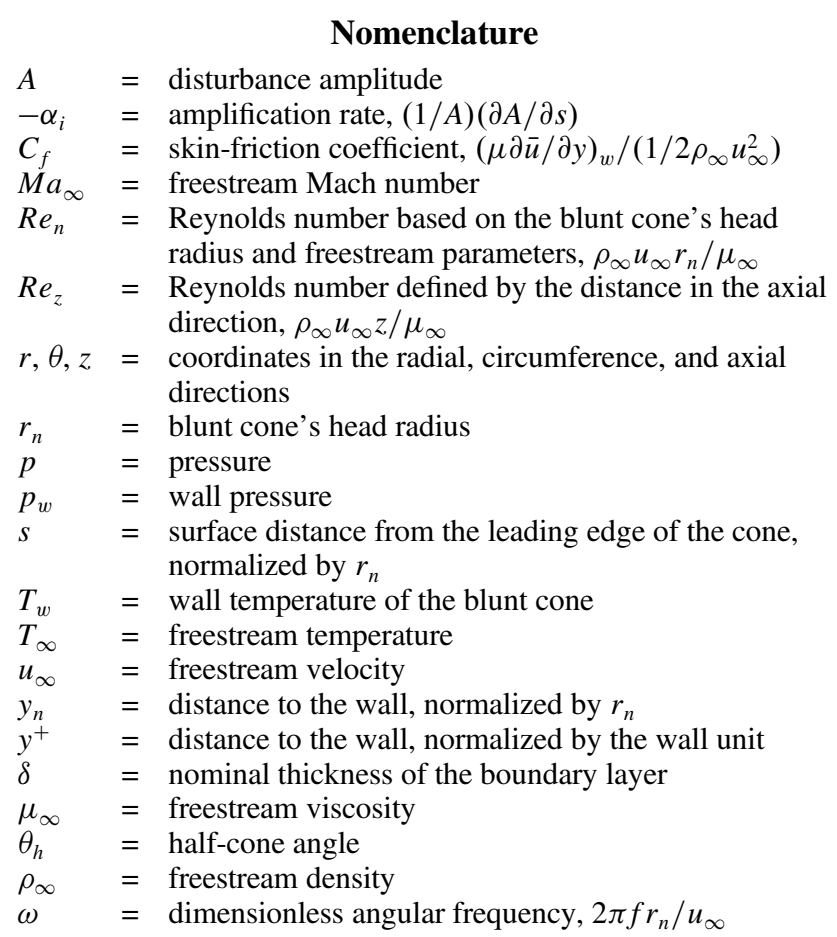

\section{Introduction}

B OUNDARY-LAYER flows over flying vehicles determine the characteristics of aerodynamic force and aerodynamic heating. It is crucial to correctly predict the laminar-turbulent transition in the boundary layers for the aerodynamic designs, thermal protections, and flying vehicle control. Because blunt or sharp cones are typical

Received 27 February 2008; revision received 5 May 2008; accepted for publication 4 June 2008. Copyright (C) 2008 by the American Institute of Aeronautics and Astronautics, Inc. All rights reserved. Copies of this paper may be made for personal or internal use, on condition that the copier pay the $\$ 10.00$ per-copy fee to the Copyright Clearance Center, Inc., 222 Rosewood Drive, Danvers, MA 01923; include the code 0001-1452/08 \$10.00 in correspondence with the CCC.

*Laboratory of Nonlinear Mechanics; lixl@lnm.imech.ac.cn. leading shapes of the supersonic and hypersonic vehicles, transition flows in the supersonic or hypersonic boundary layers around blunt cones or sharp cones are of great interest in aerospace engineering. Many flight [1] and wind-tunnel experiments [2] for boundary-layer transition of blunt or sharp cones have been conducted, with emphasis on the measurement of transition locations. Horvath et al. [2] conducted experimental investigations on 5-deg half-angle cones in a conventional wind tunnel and a quiet Mach 6 wind tunnel, and the influence of tunnel noise was studied by comparing transitiononset locations obtained from a conventional tunnel test to that obtained from a quiet-tunnel test. The comparison shows that the transition-onset Reynolds number under low-disturbance conditions was a factor of 1.3-1.6 greater than that measured in the conventional hypersonic tunnel or in the quiet-tunnel run "noisy." In addition, leading bluntness, angle-of-attack, and wall-roughness effects on transition-onset location are also discussed in report of Horvath et al. Experimental results show that leading bluntness significantly affects the transition location. The increasing of leading bluntness can retard the transition location, but the effect will be reversed when the bluntness exceeds a threshold. The effect of bluntness on the transition location is considered to associate with the entropy layer generated by the leading bow shock. The true mechanism, however, is still not clear now.

Transition mechanisms of supersonic or hypersonic boundary layers are much more complicated than those of incompressible or low-speed boundary layers. According to Mack's [3] theory, in addition to the first instability mode (counterpart of TollmienSchlichting waves), there are higher acoustic instability modes with higher frequencies in supersonic or hypersonic boundary layers. In contrast to the unstable oblique first mode in low-speed boundarylayer flow, the two-dimensional second Mack mode is dominated in hypersonic boundary layers, which has been confirmed in experiments for hypersonic boundary-layer flows over sharp or blunt cones (Stetson and Kimmel [4,5] and Maslov et al. [6] ). Stetson and Kimmel [5] carried out hypersonic boundary-layer stability experiments of a blunt cone with a 7-deg half-angle and 0-deg angle of attack (AOA), and fluctuation spectrums at different locations were measured. The experiments of Stetson and Kimmel $[\underline{4,5}]$ show that the unstable second modes are dominant disturbance waves, with significant superharmonic waves.

The transition mechanism of the supersonic or hypersonic boundary layer is not well understood yet, and no method is capable 
of predicting transition locations accurately without relying on empirical parameters. The most widely used transition prediction method is the $e^{N}$ method, which suggests that the transition occurs when the amplitude of most unstable disturbance grows $e^{N}$ times. Here, $N$ (usually between 9 and 11 for an incompressible boundary layer) is a number from the experiment. As addressed by Zhong and Ma [7], the limit of the $e^{N}$ method is that it does not consider the initial amplitude of disturbance, whereas the transition process depends on the initial disturbance. The properties of initial disturbance (including the amplitudes, frequencies, wave numbers, and other parameters) can be better understood through the study of receptivity [그, $]$ ]. Zhong and Ma [7], Ma and Zhong [9, 10], and Zhong [11] studied the receptivity of supersonic boundary layers to freestream disturbance. According to the investigations by Zhong and $\mathrm{Ma}$ [7], Ma and Zhong [9,10], and Zhong [11], there is a family of stable wave modes in the supersonic boundary layer in addition to the well-known unstable Mack modes. These stable wave modes play an important role in the receptivity process through resonant interactions with both fast sound waves and unstable Mack modes. Zhong [11] also found that freestream fast sound waves play a major role in the generation of boundary-layer stability waves, rather than the entropy waves and vorticity waves. Tumin [12] developed an instability-mode analysis tool named the multimode decomposition method and used it in the receptivity of the hypersonic boundary layer to blow-and-suction perturbation $[13,14]$. Although the $e^{N}$ method has many shortcomings, it remains one of the most powerful approaches of transition prediction. By using the $e^{N}$ method, Su and Zhou [15] calculated transition locations of the hypersonic boundary layer over a blunt cone with a freestream Mach number of 6 and a Reynolds number defined by cone radius 10,000 . The results of $\mathrm{Su}$ and Zhou show that the wall-temperature condition has a great effect on the transition location, and the second-mode waves play a dominating role in the boundary-layer transition with the isothermal wall, whereas the first mode waves dominate the transition of the boundary layer with the adiabatic-wall condition.

Because of developments in computational techniques and hardware, direct numerical simulation (DNS) is becoming a more and more important tool in the study of turbulent flows and transitions [16]. Unlike Reynolds-averaged Navier-Stokes or large eddy simulation, no artificial assumption of turbulence or transition is needed in DNS. Because of complexity, DNS of the compressible turbulent boundary layers started just recently. In the beginning, DNS cases are temporally evolving boundary layers (Guarini et al. [17] and Maeder et al. [18]) and then spatially evolving ones. Rai et al. [19] performed DNS for a spatially evolving flat-plate boundary at freestream Mach number 2.25 by using a fifth-order upwindbiased finite difference scheme for convection terms and a fourthorder central-difference scheme for viscous terms. Pirozzoli and Grasso [20] performed the same DNS case later by using a seventhorder weighted essentially nonoscillatory (WENO) scheme for convection terms and a fourth-order Pade scheme for viscous terms. They investigated the effects of the numerical scheme's resolution on the mean velocity. Compared with a temporally evolving DNS, the spatially evolving DNS does not need slow growth or extended temporal assumptions and thus minimizes any uncertainty or artificial assumption [20]. The authors previously performed direct numerical simulations of a spatially evolving boundary layer over a blunt wedge [21] with $M a_{\infty}=6$ and boundary layers over flat plates [22-24] with the freestream Mach numbers $M a_{\infty}=0.7,2.25$, and 6 . The mean pressure gradient and compressibility effects on the coherent structure were studied in these reports.

Shock waves pose severe challenge to the DNS of compressible turbulent flows (Moin and Mahes [16]). The numerical methods designed for incompressible flow become unstable in the DNS of compressible turbulence with shock waves, and negative pressure or temperature often occurs in the simulation. According to the authors' experience, even in the case without large-scale shock waves in the computing domain (such as flat-plate boundary layers), negative pressure or temperature still could occur if the Mach number is high and the numerical method is not robust. In the DNS of supersonic/ hypersonic boundary layers, negative pressure or temperature usually occurs in the location-of-transition region at the streamwise direction and occurs in the buffer layer (between the log-law region and the viscous sublayer) at the wall-normal direction, because the strongest disturbance exists in these regions. In recent years, high-order WENO schemes (Jiang and Shu [25]) have been widely implemented in the DNS of compressible turbulence. Many modified or optimized versions of WENO schemes have also been developed (Ren et al. [26], Wang and Chen [27], Martin et al. [28], and Borges et al. [29]). High-order WENO schemes show strong robustness and high resolution in the DNS of compressible turbulence. However, there are some shortcomings with this type of scheme. WENO schemes have more numerical dissipation and are computationally costly than conventional (not shock-capturing) finite difference schemes. How to construct a robust finite difference scheme with high efficiency and low numerical dissipation is still an open question.

Compared with DNS of flat-plate boundary layers, studies on the DNS of supersonic/hypersonic boundary layers over cones are seldom reported. In addition, most of the numerical studies on supersonic/hypersonic boundary-layer flow over cones take the assumption of axisymmetrical mean flow. In practice, however, the mean flows around cones are not axisymmetrical, because of the nonzero angle of attack, and the boundary-layer transition is strongly affected by this axial asymmetry. Experimental research shows that even a very small angle of attack (such as $1 \mathrm{deg}$ ) can affect the transition locations of the cone boundary layer significantly (Horvath et al. [2] and Stetson [30]). Most experimental studies show that the transition location in the leeward section (the leeward meridian plane) occurs much earlier than that in the windward section (the windward meridian plane), but the reason why transition occurs earlier in the leeward section (or delayed in the windward section) is still unclear. Crossflow is usually considered as an important reason that leads to the different transition locations between the two sections. And a simple explanation is that crossflow leads to the thickening boundary layer in the leeward section and the thinning boundary layer in the windward section, then leads to the higher growth rate of unstable waves in the leeward section, and then leads to the earlier transition in the leeward section. According to the DNS result of this research, crossflow does lead to the thickening boundary layer in the leeward section and the thinning boundary layer in the windward section. However, according to our linear stability theory (LST) analysis, the growth rate of unstable waves in the windward section is not lower than that in the leeward section. The mechanism of how crossflow affects the transition locations needs to be further studied, and more investigations need to be done to address this mechanism. Investigations based on DNS are deemed to be an important step toward understanding the mechanism of the effects of angle of attack. However, to the authors' knowledge, there is still no report for DNS of the hypersonic boundary layer over cones with angle of attack.

In this paper, the DNS of supersonic blunt-cone boundary-layer transition with a freestream Mach number of 6 and a 1-deg angle of attack was performed to study the mechanism of the transition. The frequency spectrum of disturbance waves at different locations are analyzed and compared with the LST. The different growth routes of dominating disturbance waves between the leeward and windward sections are studied and the reason why transition is delayed in the windward section is given.

\section{Flow Conditions and the Numerical Method}

Consider the supersonic boundary-layer flow around a blunt cone, as shown in Fig. 1. Flow conditions are as follows: $R e_{n}=10,000$ (where $R e_{n}=\rho_{\infty} u_{\infty} r_{n} / \mu_{\infty}$ is the Reynolds number defined by freestream parameters and blunt cone's head radius $r_{n}$ ), the freestream Mach number $M a_{\infty}=6, \mathrm{AOA}=1 \mathrm{deg}$, the half-cone angle $\theta_{h}=5 \mathrm{deg}$, the freestream temperature $T_{\infty}=79 \mathrm{~K}$, and the wall temperature of the blunted cone $T_{w}=294 \mathrm{~K}$.

Numerical simulations in this paper consist of two steps: the first step is laminar (steady) flow simulation, including the bow shock and without any perturbation, and the second step is unsteady simulation of transition flow. The steady-flow solution is used as the mean profile for LST analysis and is also used as the initial and boundary 
conditions for the unsteady simulation. The code Hoam-OpenCFD, developed by the authors, is used in this simulation. In this code, compressible Navier-Stokes equations are solved numerically by using a high-order finite difference method:

1) Convection terms are split by using Steger-Warming splitting and are discretized with a seventh-order WENO scheme [25].

2) An eighth-order central finite difference scheme is used for the viscous terms.

3) A third-order total-variation-diminishing type of Runge-Kutta method [25] is used for time advance.

The computational domain of unsteady (transition) simulation in the circumferential direction spanned from the 0-deg meridian plane to the 360-deg plane. To get high mesh resolution in the interested regions and to reduce the computing cost, computational mesh is condensed around the section $\theta=0 \mathrm{deg}$ (leeward section) or $\theta=$ $180 \mathrm{deg}$ (windward section), with a span of $45 \mathrm{deg}$ (see Fig. 2). This means that two independent computation cases are performed: the mesh for one case is condensed around the leeward section, and the mesh for another case is condensed around the windward section. From circumference two-point autocorrelations in the turbulence region, the computational domain in a 45-deg range is wide enough to ensure low two-point autocorrelations in circumference. The computational domain of unsteady (transition) simulation in the axial section is also shown in Fig. 2. The whole computational domain is located inside the bow shock (see Fig. 1). The upper boundary in the axial section is a line with a 8.5-deg tile angle to $z$ axial, and the computational domain in the wall-normal direction contains more than 10 times the boundary layer's thickness. The streamwise computational domain, mesh number, and mesh resolution (in wall unit) is shown in Table 1 , in which $z$ is the coordination in the axial direction (see Fig. 1) and the length is normalized by the cone's head radius.

Boundary conditions for simulations of unsteady flow are as follows:

1) For the inflow boundary and upper boundary (see Fig. 2), timeindependent conditions are obtained from steady simulation of the first step.

2) A nonreflecting boundary condition [31] is used on the outflow boundary in the streamwise direction.

3) A periodical boundary is used in the circumference direction $(\theta=0$ and $360 \mathrm{deg})$.

4) For the wall boundary, an isothermal wall temperature together with assumption $\partial p / \partial y_{n}=0$ is used on the wall, and a second-order one-side finite difference scheme is used to compute the wall pressure. Wall blow-and-suction perturbations are used to trigger the transition. The streamwise and circumference velocities are taken as zero on the wall, and the wall-normal velocity on the wall is

$$
v_{n}= \begin{cases}v_{\mathrm{bs}} & z_{a} \leq z \leq z_{b} \\ 0 & \text { otherwise }\end{cases}
$$

where $v_{\text {bs }}$ is the wall blow-and-suction perturbation velocity imposed on the wall in the range of $90=z_{a} \leq z \leq z_{b}=100$. In this research, both multifrequency perturbation and random perturbation are considered, and details of the perturbations can be found in Secs. III and IV.

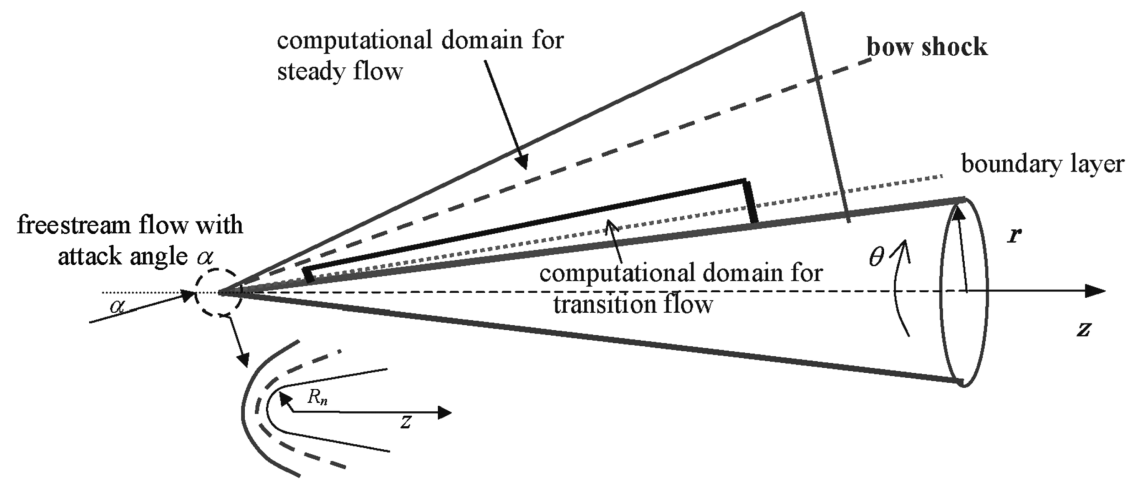

Fig. 1 Schematic of the supersonic boundary layer around a blunt cone.

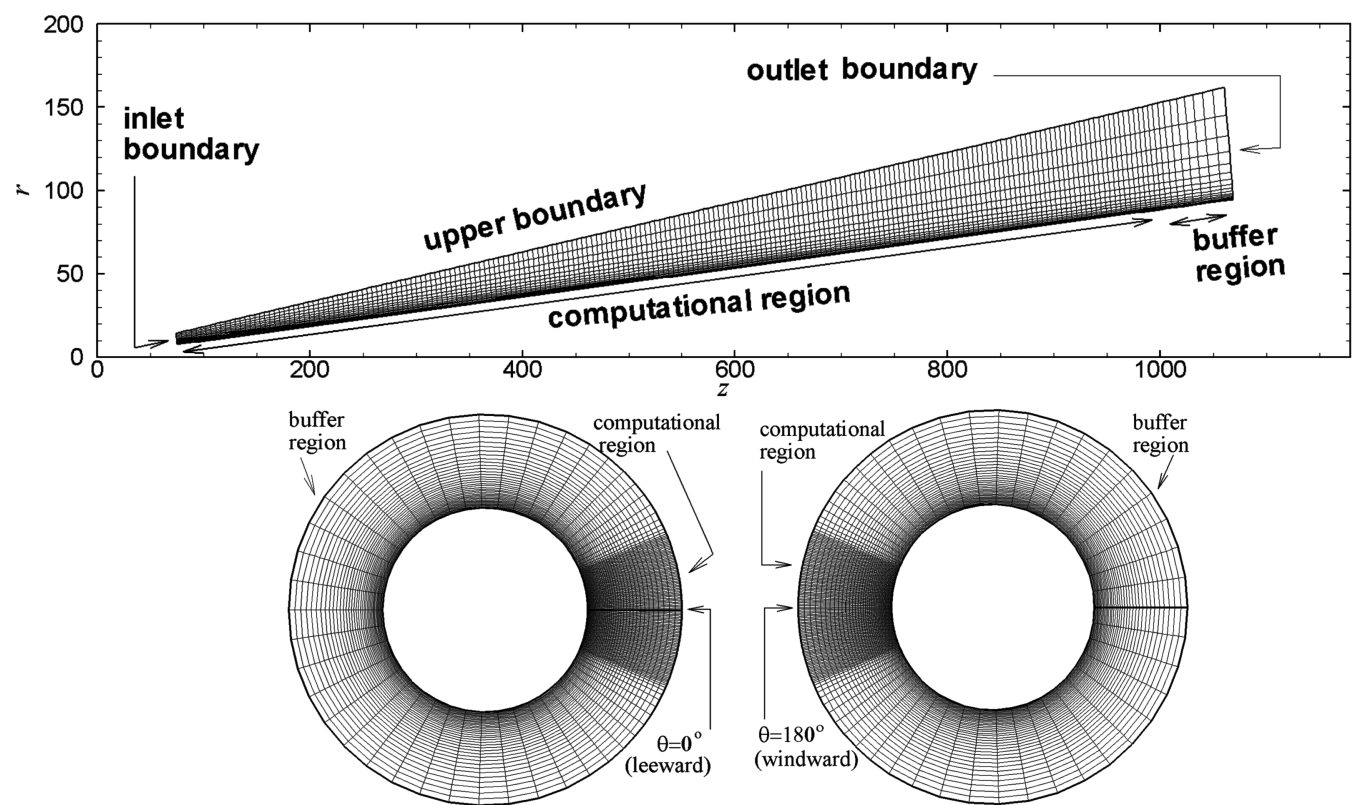

Fig. 2 Schematic of the mesh for unsteady simulation in the axial section (top) and circumference section (bottom). 
Table 1 Mesh parameters

\begin{tabular}{|c|c|c|c|c|c|}
\hline Case & Computation domain in streamwise direction & Mesh number $($ streamwise $\times$ wall normal $\times$ circumference) & $\Delta x^{+}$ & $\Delta y_{w}^{+}$ & $r \Delta \theta^{+}(z=500)$ \\
\hline Leeward & $75<z<800$ & $1800 \times 80 \times 300$ & 21.3 & 1.0 & 8.6 \\
\hline Windward & $75<z<1000$ & $2100 \times 80 \times 300$ & 23.2 & 1.0 & 8.6 \\
\hline
\end{tabular}

Because no nonreflecting or absorbing boundary conditions are used at the upper boundary, there are numerical fluctuation reflections in this boundary. However, because the upper boundary is far enough from the boundary layer (more than 10 times the boundary-layer thickness), the numerical disturbances reflected from the upper boundary are very weak in this case. In the temporal DNS of the boundary layer, with the periodical boundary condition assumed to be in streamwise direction, a well-designed nonreflecting or absorbing upper boundary condition is critical, even though the upper boundary is far enough, because reflecting waves will be amplified repeatedly through the streamwise periodic condition. For the spatial DNS in this research, reflected waves of the upper boundary are not so serious if the upper boundary is far enough (Adams [32] and Egorov et al. [33]). Moreover, as addressed by Adams [32], the mesh coarsening toward the upper boundary lets the numerical diffusion of the discretization scheme act as a filter that removes spurious wave reflections from the boundary. In our DNS, the numerical dissipation on the very large grid space near the upper boundary absorbs the reflection waves. The authors tested the reflection waves from the upper boundary and found that the fluctuation near the upper boundary layer is very small and the computational domain is not polluted by nonphysical reflection waves from the upper boundary (see Sec. VI).

The DNS was performed on supercomputers DeepComp 6800 in the Supercomputing Center at the Chinese Academy of Sciences and Dawning 4000A in the Shanghai Supercomputing Center; 128-256 CPUs were used for the DNS cases.

\section{Code Validation}

\section{A. Comparison Between Different Codes}

To test our code, we compare the results of our code with the results of another code that uses a different numerical method. Figures $\underline{3 a}$ and $\underline{3 b}$ show the steady wall pressure in the leeward section and windward section, respectively. The solid line denotes the current computation and the symbol denotes the corresponding results from a shock-fitting computational fluid dynamics code [34]. The horizontal coordinate $s$ denotes the cone surface location, and the relation between $s$ and $z$ shows as follows:

$$
s= \begin{cases}\cos ^{-1}(-z) & z<-\sin \left(\theta_{h}\right) \\ \left(z+\sin \left(\theta_{h}\right)\right) /\left(\cos \theta_{h}\right) \approx 1.0038 z+0.0875 & z>-\sin \left(\theta_{h}\right)\end{cases}
$$

The agreement between the two results validates our code. Because of the small attack of angle, the difference between wall pressure in the leeward section and that in the windward section is not obvious (the maximum difference is about $3 \%$ of the stagnation pressure).

\section{B. Comparison with the Axisymmetrical Simulation}

To further verify our code, we simulate steady flow of Mach 7.99 flow over a blunt cone with a 0 -deg angle of attack. Flow conditions of this case are

$$
\begin{gathered}
M a_{\infty}=7.99, \quad R e_{n}=\frac{\rho_{\infty} u_{\infty} r_{n}}{\mu_{\infty}}=33,449, \quad \theta_{h}=7 \mathrm{deg} \\
\text { AOA }=0 \mathrm{deg}, \quad \text { adiabatic wall, } \quad T_{\infty}=54.474 \mathrm{~K}
\end{gathered}
$$

Zhong and Ma [7] gave a high-quality numerical solution of this steady boundary-layer flow by using Zhong's [35] high-order shockfit method, and their numerical solution is well validated compared with both experimental results (Stetson et al. [36]) and other numerical results (Herbert and Esfahanian [37]). To test our code, we compare our result with the results of Zhong and Ma [7]. Our steady numerical simulation is carried out on a mesh with 1600 (streamwise) $\times 160$ (wall normal) $\times 8$ (circumference) grid points. Because the attack of angle is zero, the steady flow is two-dimensional (axisymmetrical), and the mesh number in circumference does not affect the numerical accuracy. Figures $\underline{4}$ and $\underline{5}$ show steady pressure and temperature distributions along the cone surface, respectively. Those two figures show very good agreement between our numerical results and the results of Zhong and $\mathrm{Ma}$ [7]. Unlike wall pressure, the numerical result of wall temperature is very sensitive to numerical accuracy, especially to the accuracy of boundary conditions and boundary schemes. Figure 6 shows the good agreement in tangential velocity profiles across the boundary layer at a surface location of $s=94$.

\section{Steady-Flow Profiles and the Crossflow}

Figure $\underline{7}$ shows steady profiles of density, stream velocity, and temperature in the leeward section and in the windward section at the locations $z=100$ and 300 . This figure shows a very big difference of mean flow profiles between those in the leeward section and in the windward section, even when the angle of attack is only $1 \mathrm{deg}$. The thickness of the boundary layer in the leeward section is more than twice that in the windward section.

Figure 8 shows the streamline patterns in the surface $k=10,20$, and 40, where $k=10$ means the 10th grid from the wall (the wall is $k=1$ ). The distances to the wall at $z=80$ are $k=10$ and $y_{n} \approx 0.1$ (about $5 \%$ of $\delta$ ), $k=20$ and $y_{n} \approx 0.24$ (about $12 \%$ of $\delta$ ), and $k=40$ and $y_{n} \approx 0.62$ (about $31 \%$ of $\delta$ ), where $y_{n}$ is the distance to the wall
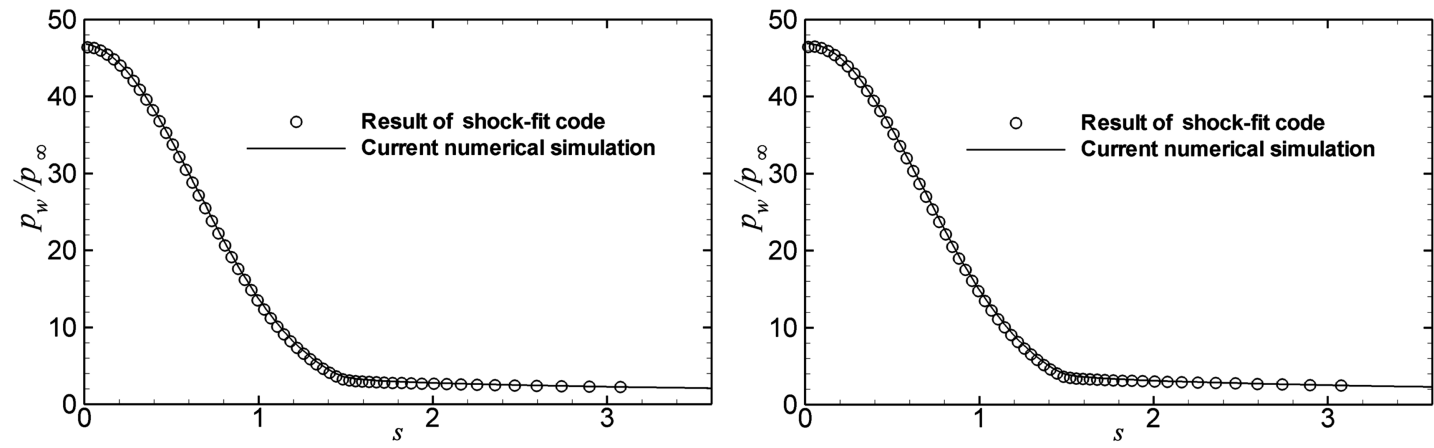

a)

b)

Fig. 3 Steady wall pressure in the leeward section (left) and in the windward section (right). 


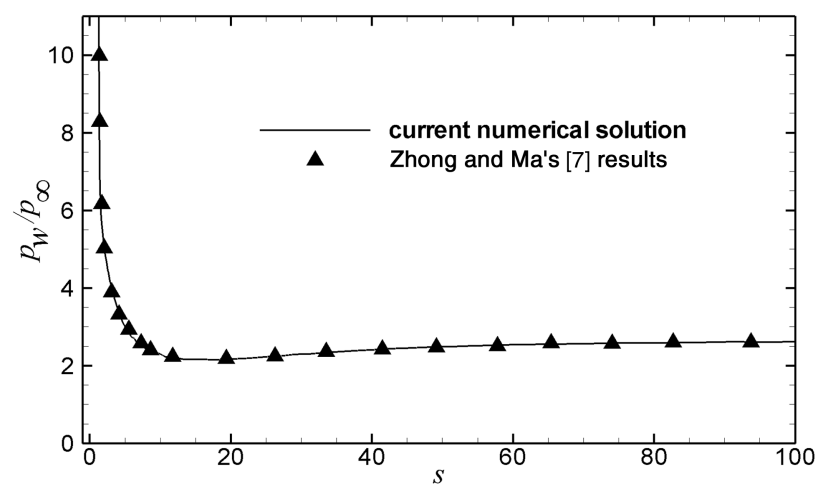

Fig. 4 Steady wall pressure compared with the results of Zhong and Ma [7].

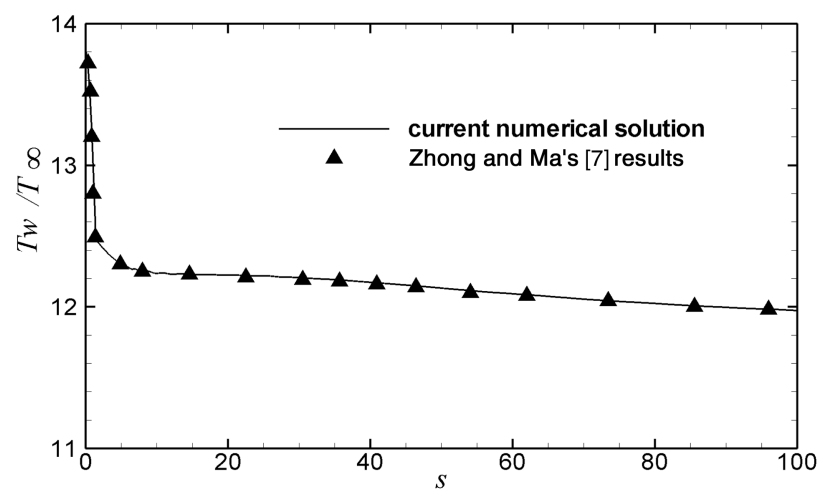

Fig. 5 Steady wall temperature compared with the results of Zhong and Ma [7].

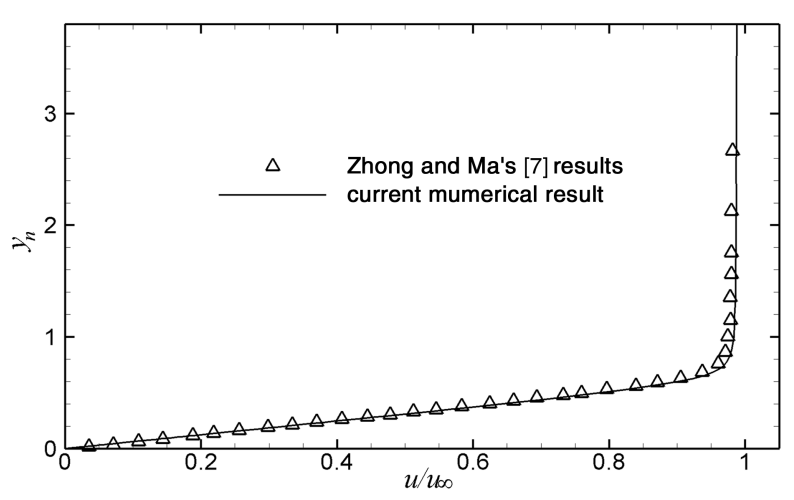

Fig. 6 Tangential velocity profile along the wall-normal direction at $s=94$ compared with the results of Zhong and Ma [7].

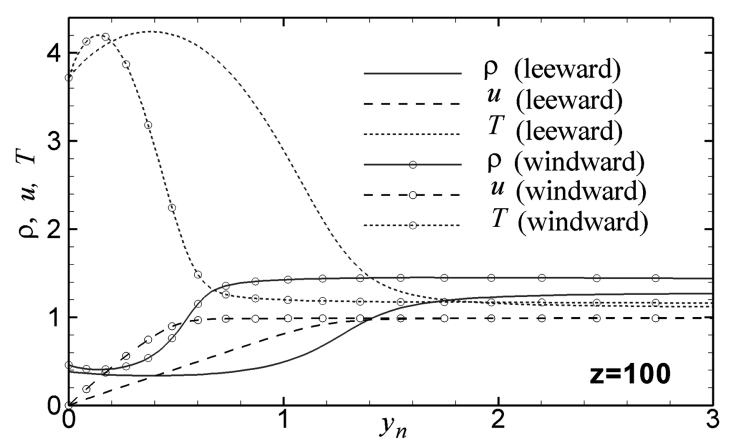

(normalized by the head radius) and $\delta$ is the nominal thickness of the local boundary layer. The streamlines in Fig. 8 show very clear crossflow in the bottom region of the boundary layer. And the crossflow is thought of as the main reason that leads to different profiles of boundary layers between the leeward section and the windward section.

\section{Linear Stability Theory Analysis}

Using the mean profiles obtained from the three-dimensional steady numerical solution, LST analysis is conducted to study the instability modes. According to LST, disturbance waves have the following expression:

$$
\mathbf{f}^{\prime}=\hat{\mathbf{f}}\left(y_{n}\right) e^{i\left(\alpha s+\beta z^{\prime}-\omega t\right)}
$$

where $\mathbf{f}^{\prime}=\left[\rho^{\prime}, u^{\prime}, v^{\prime}, w^{\prime}, T\right]^{T}$ is the disturbance of flow and $\hat{\mathbf{f}}\left(y_{n}\right)$ is its eigenfunction; $\alpha, \beta$, and $\omega$ are the streamwise wave number, spanwise wave number, and frequency of disturbance waves, respectively; $z^{\prime}$ is the local spanwise coordinate; $-\alpha_{i}=-\operatorname{Im}(\alpha)$ is the growth rate of the disturbance wave; and $-\alpha_{i}>0$ means an unstable wave. The growth rate is a function of $\omega$ and $\beta$. In our LST analysis, a finite difference code SAYR [15] is used. In the parameter plane $(\omega, \beta)$, there are some distinct regions that satisfy the unstable condition $-\alpha_{i}(\omega, \beta)>0$. The region with the lowest $\omega$ relates to the first unstable mode and the region with higher $\omega$ relates to the second mode. The second mode's growth rate reaches its maximal value when $\beta=0$, and so the second mode is thought of as a twodimensional mode, whereas the first mode is a three-dimensional mode. By searching in the parameter plane $(\omega, \beta)$, the growth rates $-\alpha_{i}$ of the most unstable first and second modes are given in Table 2 , where $z$ is the axial location normalized by the nose radius. This table shows that the growth rate of the unstable second mode is much higher than that of the first mode, especially in the windward section. The higher growth rate indicates that the second mode will dominate the transition.

Table 2 shows that the growth rate of the unstable second-mode wave in the windward section is not lower than that in the leeward section. And the delayed transition in the windward section cannot be interpreted simply by the lower growth rate of the unstable waves. The reasons why transition is delayed in the windward section need to be further studied.

\section{Transition Triggered by Weak Random Blow-and-Suction Disturbance}

DNS of transition flow triggered by random blow-and-suction disturbance is conducted. On the wall, the wall-normal velocity is set to be random numbers between $-\varepsilon$ and $\varepsilon$ in the range of $90=z_{a} \leq$ $z \leq z_{b}=100$ and 0 outside this region. The disturbance amplitude is set to be $\varepsilon=0.01$. This perturbation is spatially random-distributed and stays temporally constant.

Figures $9 \mathrm{a}$ and $9 \mathrm{~b}$ show the skin-friction coefficient $C_{f}$ along the streamwise direction in the leeward section and in the windward section, respectively. The solid line denotes the result of the DNS and

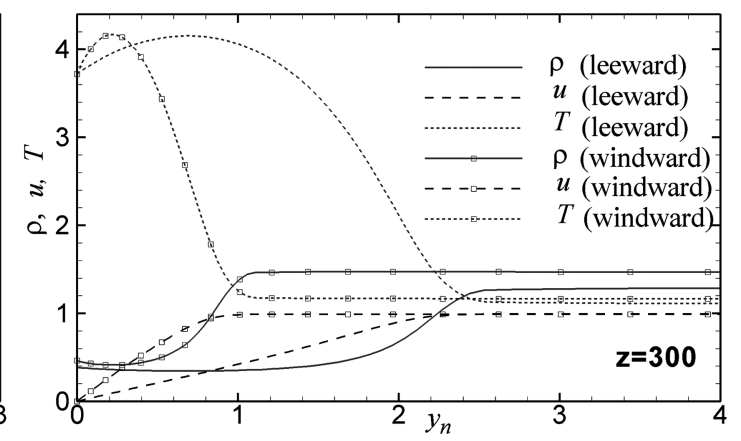

Fig. 7 Profiles of density, streamwise velocity, and temperature in the leeward section and in the windward section; $z=100$ (left) and 300 (right). 

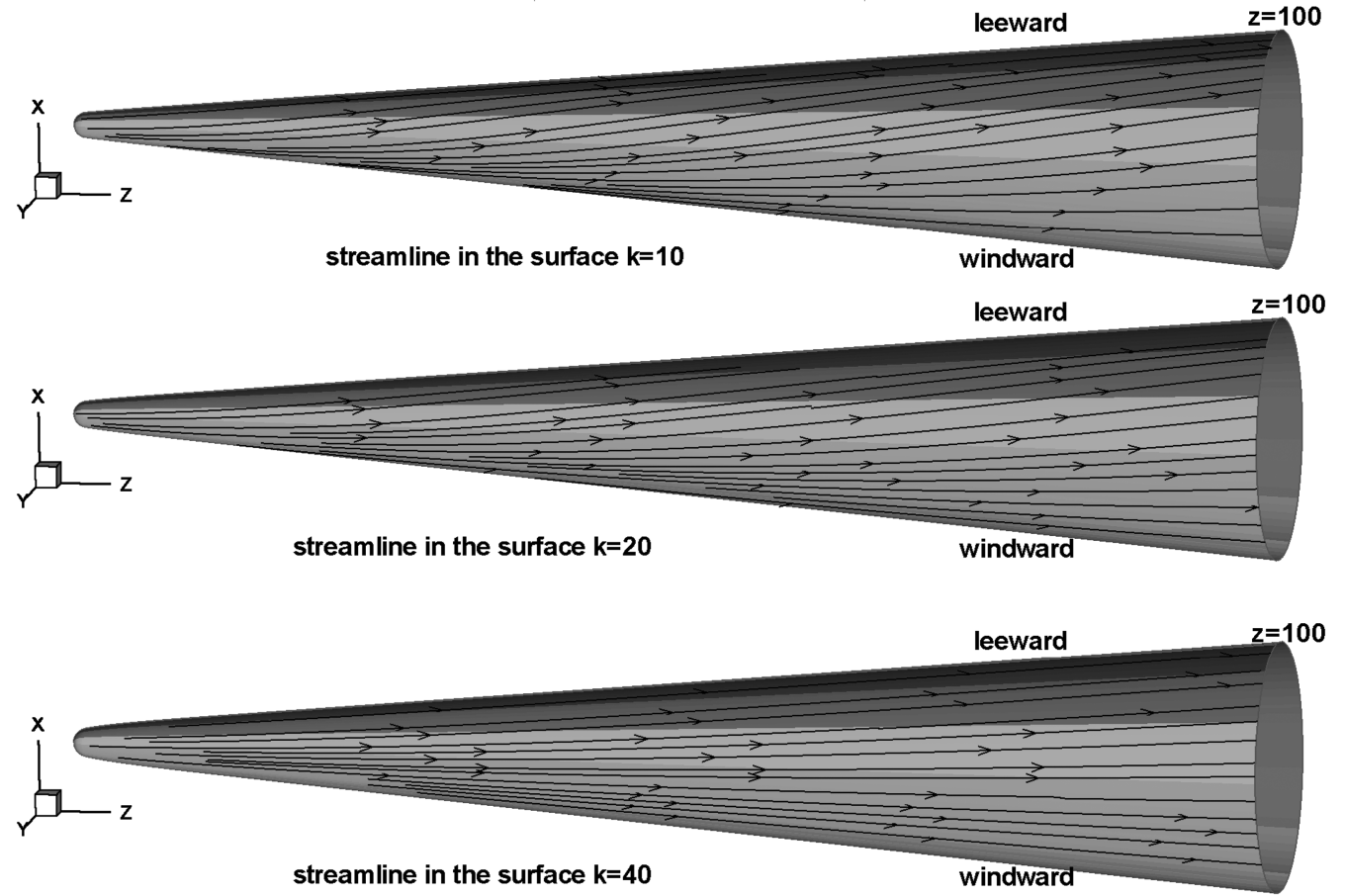

Fig. 8 Streamline patterns in the surface $k=10,20$, and 40 (distances to the wall at $z=80$ of $0.05 \delta, 0.12 \delta$, and $0.31 \delta$, respectively).
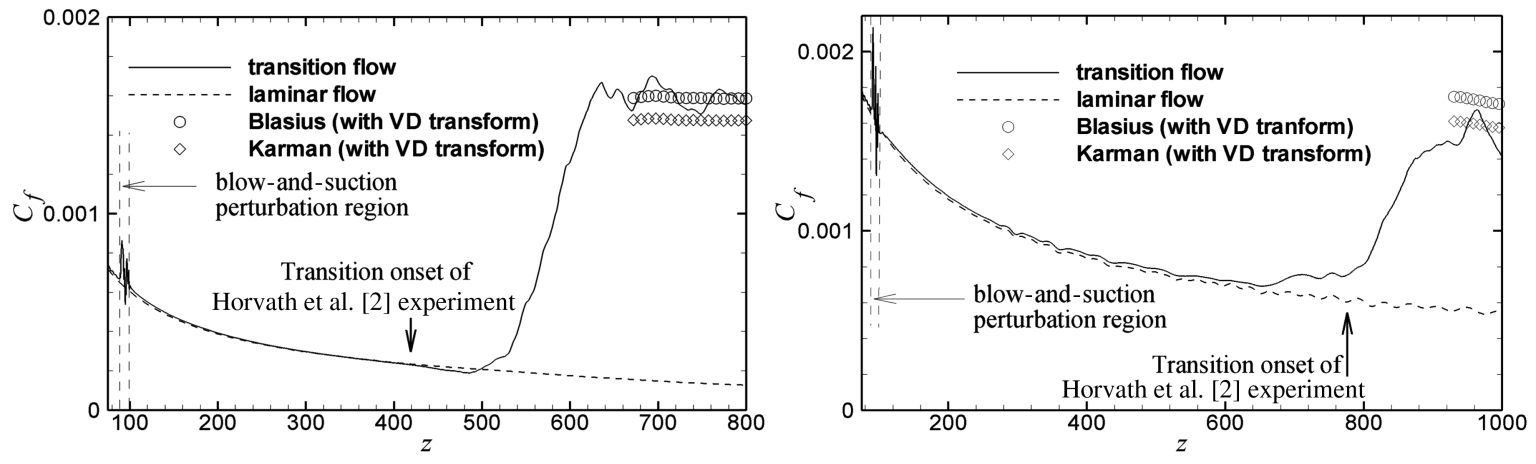

a)

b)

Fig. 9 Distribution of $C_{f}$ in the leeward section (left) and windward section (right).

the dashed line denotes the result of the laminar flow. The horizontal coordinate $z$ denotes the axial location (see Fig. 1) normalized by the nose radius. The detach location between the solid line and dashed line in Fig. 9a denotes that transition occurs at about $z \approx 500$ (transition onset) or $R e_{z} \approx 5 \times 10^{6}$. The transition peak is located at $z \approx 630$ or $R e_{z} \approx 6.3 \times 10^{6}$ in the leeward section. Figure $9 \mathrm{~b}$ denotes that transition onset is located at about $z \approx 750$ or $R e_{z} \approx$ $7.5 \times 10^{6}$ and transition peak is located at $z \approx 960$ or $R e_{z} \approx 9.6 \times$ $10^{6}$ in the windward section. In Fig. 9, the theoretical values of turbulent skin-friction coefficients are also plotted, and this figure shows that our numerical result agrees well with the theoretical result. Here, the theoretical values are computed by using KarmanSchoenherr and Blasius formulations with Van Driest (VD) II transformation (see Pirozzoli and Grasso [20] for details of the theoretical formulas).

Table 2 Growth rates $-\alpha_{i}$ of the most unstable LST modes

\begin{tabular}{ccccc}
\hline \hline & \multicolumn{2}{c}{ Leeward } & \multicolumn{2}{c}{ Windward } \\
$z$ & First mode & Second mode & First mode & Second mode \\
\hline 200 & 0.011 & 0.052 & 0.0025 & 0.057 \\
400 & 0.012 & 0.043 & 0.0038 & 0.051 \\
600 & 0.013 & 0.038 & 0.0036 & 0.046 \\
\hline \hline
\end{tabular}

Horvath et al. [2] reported the experimental results for transition locations of a Mach 6 flow over cones or flared cones with a 5-deg half-cone angle and with different bluntnesses, angles of attack, and Reynolds numbers. The report of Horvath et al. includes data obtained in the NASA Langley Research Center's Mach 6 lowturbulence tunnel. Transition locations obtained in low-turbulence tunnels are closer to those of an actual (flight) situation than those obtained in conventional tunnels; therefore, transition locations obtained in low-turbulence tunnels are more valuable for the design of hypersonic vehicles. From the experimental results of Horvath et al., it can be deduced that the transition-onset Reynolds numbers should be $R e \approx 4.2 \times 10^{6}$ in the leeward section and $R e \approx 7.8 \times 10^{6}$ in the windward section for the quiet-tunnel test of a blunt cone with the same half-cone angle ( $5 \mathrm{deg})$, the same angle of attack ( $1 \mathrm{deg})$, the same freestream Mach number $(M a=6)$, and nearly the same bluntness as in our numerical simulation. Our numerical result (transition-onset Reynolds numbers of $R e \approx 5 \times 10^{6}$ in the leeward section and $R e \approx 7.5 \times 10^{6}$ in the windward section) agrees well with the result of the Horvath et al. quiet-tunnel test.

\section{A. Transition Flow Visualization}

Figure 10 shows the distribution of instantaneous density in the boundary layer of the leeward section. The density distribution in the 


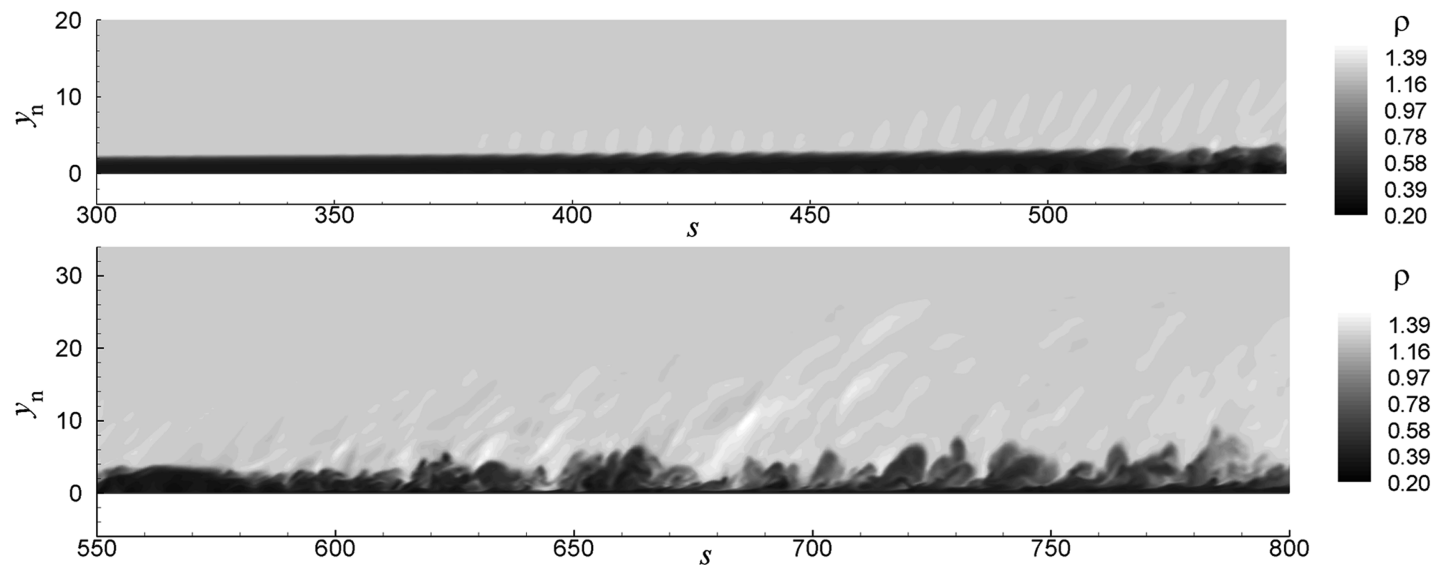

Fig. 10 Distribution of density in the boundary layer of the leeward section.
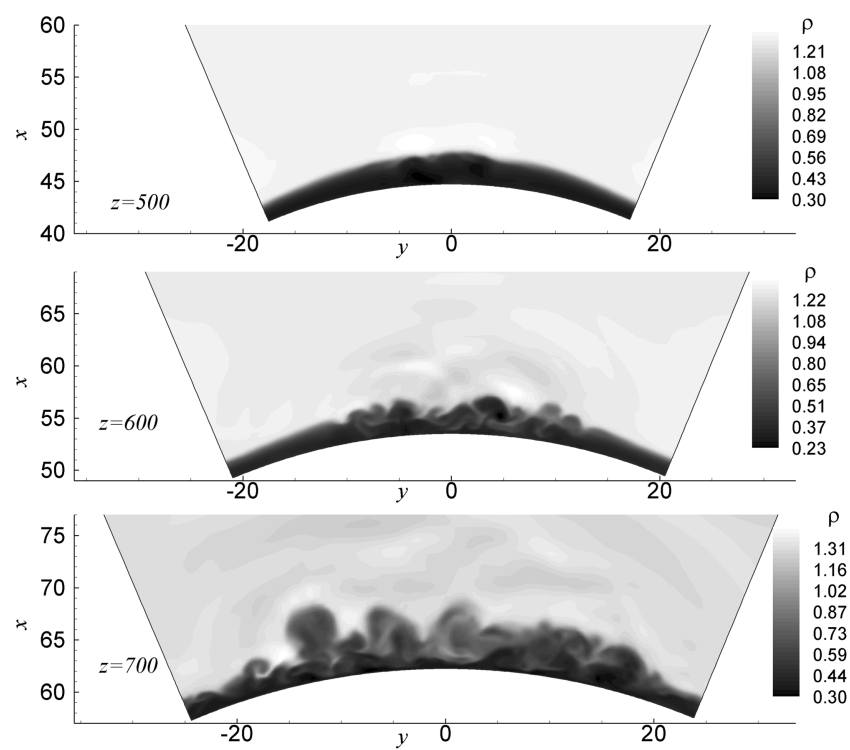

Fig. 11 Distribution of density in the boundary layer at three wallnormal surfaces.

boundary layer stays laminar in the range of $z<370$ and disturbance waves can be found in the range of $370<z<500$. The strong disturbance waves occur in the range of $500<z<550$ and the density distribution became turbulent at $z>600$. Figure 11 shows the instantaneous density distribution in three wall-normal surfaces (the surfaces normal to the generating line of the cone). The intersection of these three surfaces to the wall are located at $z=500$, 600, and 700, respectively. The spanwise (circumference) visualization region of this figure is $-22.5 \mathrm{deg}<\theta<22.5 \mathrm{deg}$ ( $\theta=0 \mathrm{deg}$ is the leeward section and $\theta=180 \mathrm{deg}$ is the windward section). Notable disturbance waves are observed near the leeward section at $z=500$, and the disturbance range extends to about $30 \mathrm{deg}$ ( $-15 \mathrm{deg}<\theta<15 \mathrm{deg})$ at $z=600$ and then to the whole visualization range at $z=700$. This figure also shows highly intermittent characters of the outer layer of the boundary layer.

Figures $\underline{12}$ and 13 show contours of the density disturbance $\rho^{\prime}$ in a wall-normal surface $z=700$ and in the leeward section, respectively, where $\rho^{\prime}=\rho-\bar{\rho} ; \rho$ and $\bar{\rho}$ are instantaneous and time-averaged density, respectively. In Figs. 12 and 13, 100 uniform levels are plotted from the minimal value to the maximal value, and a one-dimensional distribution of $\rho^{\prime}$ on the line $\theta=0 \mathrm{deg}$ on the surface $z=700$ is also plotted in Fig. 12. These two figures show that disturbance is very small near the upper boundary and that the computational domain is not polluted by nonphysical reflection waves from the upper boundary, which indicates that the upper boundary condition is suitable.

Figure 14 shows instantaneous tangential (streamwise) velocity $u$ at a wall-parallel section (distance to the wall $y_{n}=0.1$ or $y_{n}^{+} \approx 5$ ) around the leeward section for the visualization ranges of $200<$ $z<800$ and $-22.5 \mathrm{deg}<\theta<22.5 \mathrm{deg}$. The disturbance waves stay quasi-two-dimensional in the range of $z<450$ and become strong three-dimensional waves in the range of $500<z<580$. Associated with the development of the unstable Mack second mode, cell-like patterns are also found in the range of $500<z<580$. Such cell-like patterns have already been observed in the hypersonic transition boundary-layer experiments around a blunt cylinder at the Mach number $M a=8.9$ by Fiala et al. [38] and in the DNS of the hypersonic flat-plate boundary layer at $M a=6$ by Krishnan and Sandham [39]. Figure 14 also shows that streamwise streaks are formed in the range of $\overline{580}<z<700$ and become very clear in the range of $z>700$.

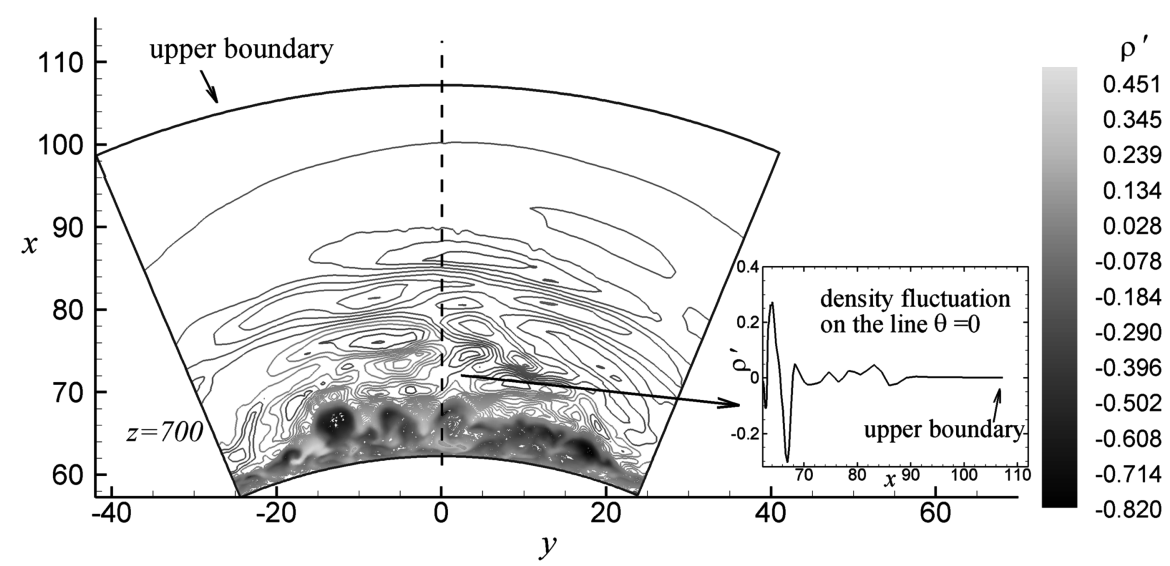

Fig. 12 Contours of the density fluctuation $\rho^{\prime}$ in a wall-normal surface $z=700$, together with one-dimensional distribution of $\rho^{\prime}$ on the line $\theta=0^{\circ}$. (100 uniform levels are set from the minimal value to the maximal value). 

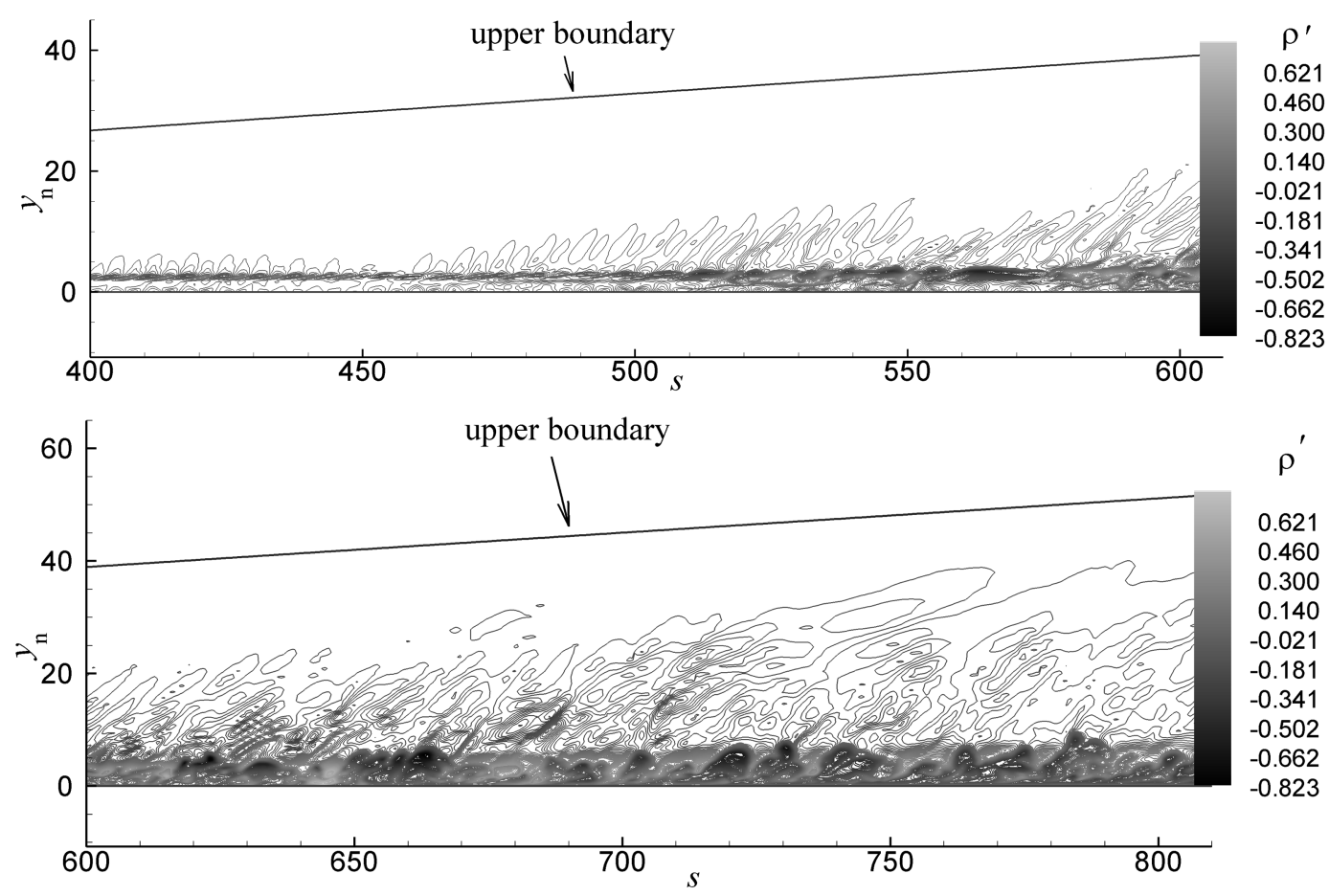

Fig. 13 Contours of the density disturbance $\rho^{\prime}$ in the leeward section. (100 uniform levels are set from the minimal value to the maximal value).

Figure 15 shows instantaneous streamwise velocity $u$ at a wallparallel section (distance to the wall $y_{n}=0.1$ ) around the windward section in the ranges of $400<z<1000$ and $157.5 \mathrm{deg}<\theta<$ $202.5 \mathrm{deg}$. The disturbance waves stay quasi-two-dimensional in the range of $z<700$ and become strong three-dimensional disturbance waves in the range of $700<z<850$. Streamwise streaks are formed in the range of $850<z<950$ and become very clear in the range of $z>950$. Different disturbance wave structures between Figs. 14 and 15 indicate that the transition mechanism is different between the windward section and the leeward section. Because the main propose of paper is to answer the question why transition occurs earlier in the leeward section or transition is delayed in the windward section, the authors pay more attention to the growth of unstable waves in the linear stage. The nonlinear mechanism of transition will be studied in the near future.

\section{B. Disturbance Waves in the Leeward Section}

We record the time history of pressure and velocity fluctuations at several fixed points in the leeward and windward sections for frequency spectrum analysis. Figures $16 \mathrm{a}-16 \mathrm{f}$ show the frequency spectrums of streamwise velocity fluctuation $u^{\prime}$ at the six points located in the boundary layer of the leeward section, where the dimensionless angular frequency $\omega$ is defined by $\omega=2 \pi f r_{n} / u_{\infty}$ and $f$ denotes dimensional frequency. The normal distances of these six points to the wall are $y_{n}=1.4 \sim 2.7$ (normalized by the nose radius), which are approximately $0.6-0.7$ times the local boundary layer's thickness. Eigenfunctions of LST show that the unstable first and second modes both have significant streamwise velocity components at these locations, and thus streamwise velocity fluctuations recorded at these points contain effects of both the first and second modes.

Figure 16a shows the wide-bandwidth frequency spectrum of streamwise velocity fluctuation at the point located at $z=100.3$, which is located just downstream of the perturbation region, and disturbance waves here can be deemed as initial disturbance waves for the transition. The amplitude stays at $\varepsilon \approx 1 \sim 3 \times 10^{-5}$ in the low-frequency range $\omega<2$, decays in the range $\omega>2$, and stays at about $10^{-6}$ in the high-frequency range $\omega>4$. From numerical analysis, it can be seen that the initial disturbance in the boundary layer $(z \approx 100)$ includes a wide-frequency bandwidth of LST modes at the level of $10^{-5}$. In the linear stage, these modes develop independently. Stable waves will decay to zero, and unstable waves are amplified. Figure $16 \mathrm{~b}$ shows the spectrum at $z=200$, where the amplitude of low-frequency $(\omega<1.5)$ components grows significantly, but the amplitude of high-frequency range does not grow yet. At $z=300$ (see Fig. 16c), several dominating frequencies are formed. The most dominating frequency is $\omega=0.9$, which is in the unstable-frequency range of the second mode (see the neutral stability curve of LST discussed later in this section). The amplitude of this wave is about $\varepsilon \approx 5.5 \times 10^{-4}$. In addition to this frequency, there are some other frequency peaks in the low-frequency region $(\omega<0.6)$, which is in the unstable-frequency range of the first mode, with an amplitude of around $\varepsilon \approx 4 \times 10^{-4}$.

At $z=400$ (see Fig. 16d), the dominating frequency becomes $\omega=0.79$, with a much higher amplitude of peak $(\omega=0.79)$ than that of other frequency peaks. Because the frequency of this wave is in the unstable-frequency range of the second mode, this wave is a secondmode disturbance wave. We compare the disturbance waves between DNS and LST mode to verify this, as shown in Fig. 17. The symbols in Fig. 17 denote the disturbance of streamwise velocity obtained from DNS: $u^{\prime}\left(y_{n}\right)=u\left(y_{n}\right)-\bar{u}\left(y_{n}\right)$ at $t=3000.4$ and $z=400$ for the leeward section from DNS, where $u\left(y_{n}\right)$ denotes instantaneous streamwise velocity, $\bar{u}\left(y_{n}\right)$ denotes time-averaged streamwise velocity, and $y_{n}$ denotes the distance to the wall normalized by the cone's head radius. The solid line in Fig. 17 denotes LST streamwise velocity disturbance: $u^{\prime}\left(y_{n}\right)=0.033 \times \overline{R e}\left(\hat{u}\left(y_{n}\right) e^{i \varphi}\right)$, where $\hat{u}\left(y_{n}\right)$ denotes the second mode's eigenfunction at $\omega=0.79$ and $\varphi=0.7$ (phase angle), and $\operatorname{Re}(a)$ denotes the real part of complex number $a$. This figure shows generally good agreement between the disturbance from DNS and the second-mode disturbance from LST, which means that the second-mode wave is the dominating disturbance in the DNS. There is still some difference between the DNS and LST results in Fig. 17, and the reason is mainly because the fluctuation of LST is a single wave (the most dominated wave); however, fluctuation of DNS is an integration of all disturbance waves, including the most dominated wave and other waves. In addition, another reason is that LST takes the assumption of parallel mean flow and linear disturbance, but there are no such assumptions in DNS.

Figure $16 \mathrm{e}$ shows that at $z=500$, the dominating second mode's amplitude grows to $\varepsilon \approx 0.025$ and the frequency is $\omega=0.75$, which means that the disturbance is strong enough for nonlinear breakdown. Figure 16e also shows a very strong peak at very low frequency $\omega \approx 0.04$. The nonlinear effects are very obvious at the 

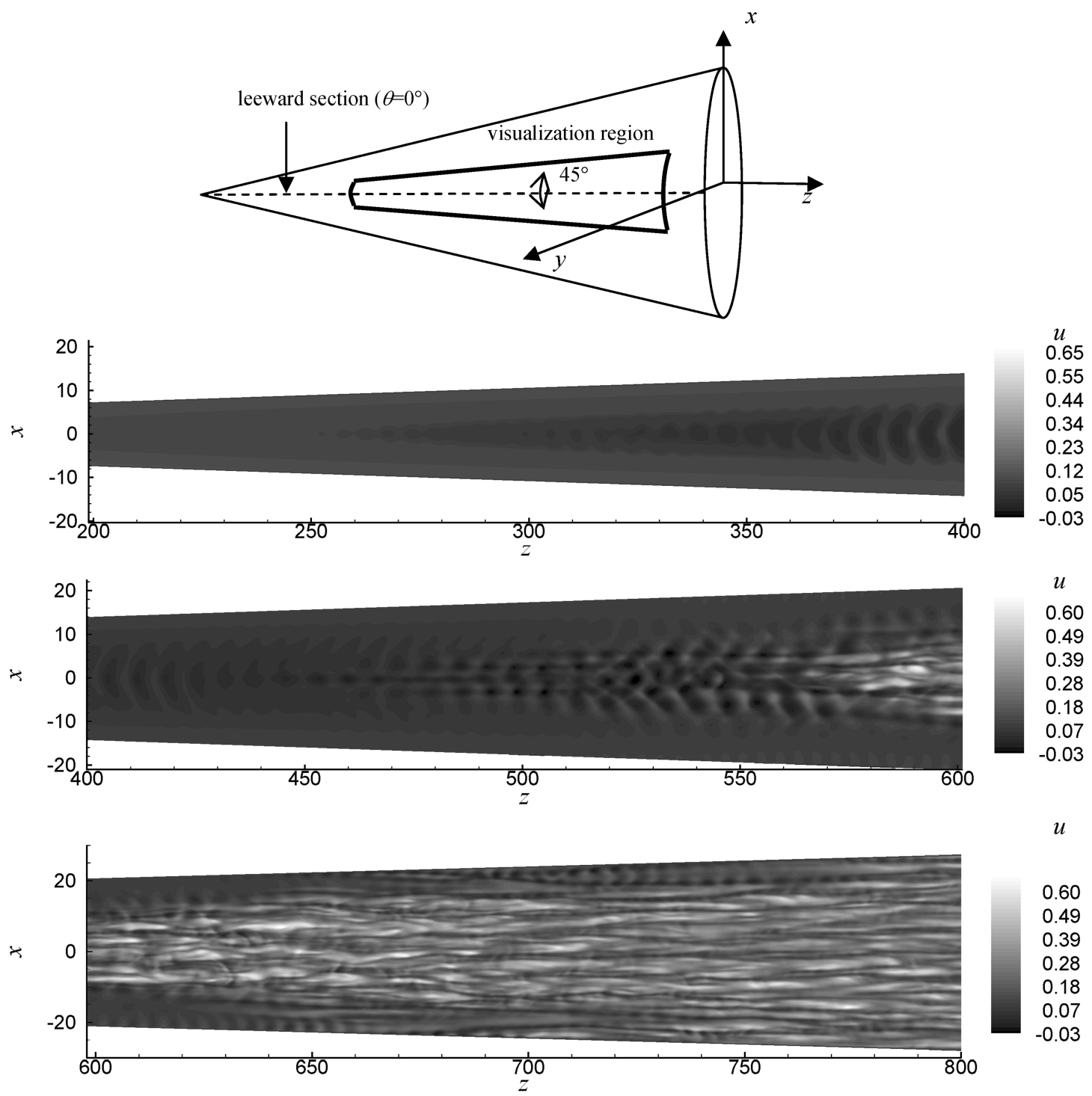

Fig. 14 Streamwise velocity at a wall-parallel section $\left(y_{n}=0.1\right)$, around leeward section.

start point of transition (close to $z=500$ ), and the rapid growth of the low-frequency disturbance is related to nonlinear mechanisms. The mechanism of rapid growth for disturbance waves during the nonlinear stage is beyond the scope of this paper, and the authors will leave it to future study. Figure $16 \mathrm{f}$ shows the wide-bandwidth spectrum at $z=700$, where transition is happening.

Figure 18 shows the frequency spectrum of pressure fluctuations at six points on the wall of the leeward section. Because the second mode has a strong pressure fluctuation component on the wall but the first mode's pressure fluctuation component on the wall is very weak, the pressure fluctuation is mainly affected by the second mode. From this figure, the frequency of the dominated second modes at different cone surface locations can be detected. This figure shows a dominating frequency $\omega=0.72$ at the location of $z=500$ (the start location of transition), and the wave with the frequency $\omega=0.72$ is deemed to be the dominating disturbance wave that triggers the transition.

Figure 19 shows the neutral stability curve obtained by LST based on the laminar numerical solution as the basic solution and the evolution route of the dominating disturbance waves in the leeward section. The symbols indicate dominating frequencies of the secondmode disturbance waves obtained from data of DNS, and the dashed line (growth route of $\omega=0.72$ ) denotes the growth route of the dominating disturbance wave that triggers the transition. Figure 20 shows the amplitude distributions of wall-pressure perturbations of different frequencies along the cone surface in the leeward section. An exponential growth of the dominating disturbance wave $(\omega=0.72)$ can be found in the range of $200<z<500$ and the growth rate approximately stays as a constant at this range. From $z=200$ to 500 , the amplitude of the dominate disturbance wave is magnified about 15,000 times, with a mean growth rate $\bar{\alpha}=\ln 15,000 /(500-200) \approx 0.032$.

\section{Disturbance Waves in the Windward Section}

Figures 21a-21i show the frequency spectrums of streamwise velocity fluctuations in nine points located in the boundary layer of the windward section. The distances of these nine points to the wall are approximately $0.6-0.7$ times the local boundary layer's thickness $\delta$. Figure 21a shows the frequency spectrum at $z=100.1$ locating just downstream of the perturbation region. It can be deemed as the spectrum of initial disturbance. Figure 21 a shows a wide-bandwidth spectrum such as the initial spectrum of the leeward section. The amplitude of the spectrum stays at $\varepsilon \approx 1 \times 10^{-5}$ to $3 \times 10^{-5}$ in the low-frequency range $\omega<2.5$, decays when $\omega>2.5$, and stays at approximately $10^{-6}$ in the high-frequency range $\omega>4$. At $z=200$ (Fig. 21b), the amplitude of the low-frequency range $\omega<0.6$ waves grows to $5 \sim 8 \times 10^{-5}$, whereas the amplitude of high-frequency components decay to $1 \sim 4 \times 10^{-6}$. At $z=300$ (Fig. 21c), the local dominating frequencies are in the low-frequency region, and the frequency spectrum shows three peaks in the frequency region $\omega=0.1-0.3$ with the amplitude of $2 \times 10^{-4}$. The frequencies of these three peaks are located in the unstable-frequency range of the first mode. Figure $21 \mathrm{c}$ shows that there is another frequency peak in 

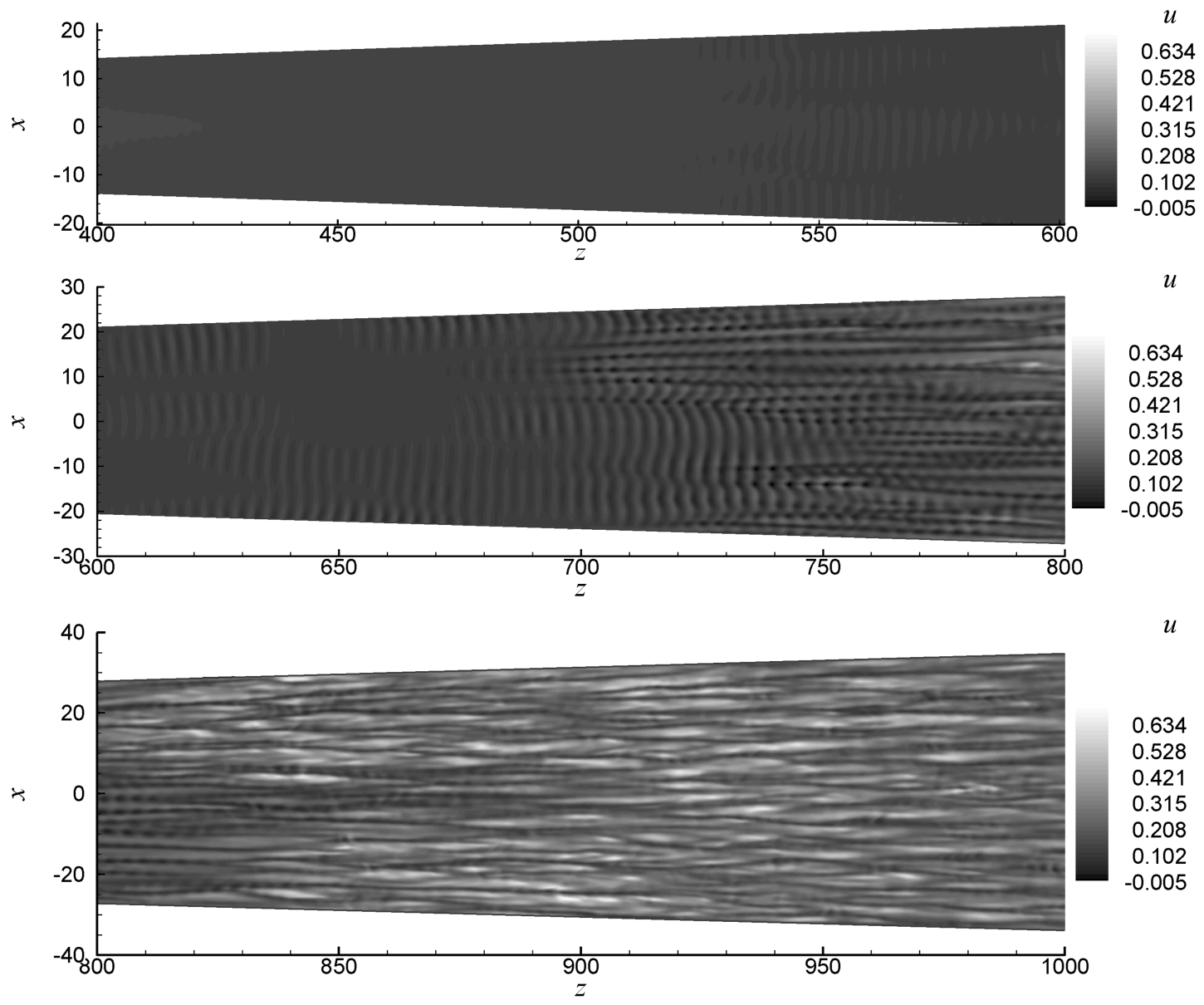

Fig. 15 Streamwise velocity at a wall-parallel section $\left(y_{n}=0.1\right)$, around windward section.

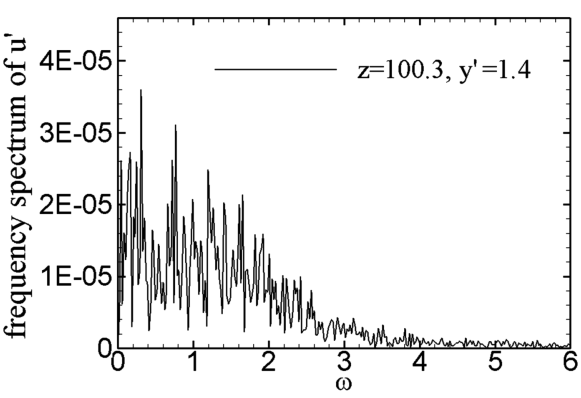

a)

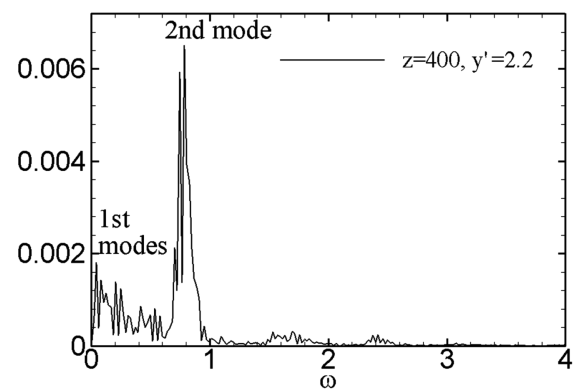

d)

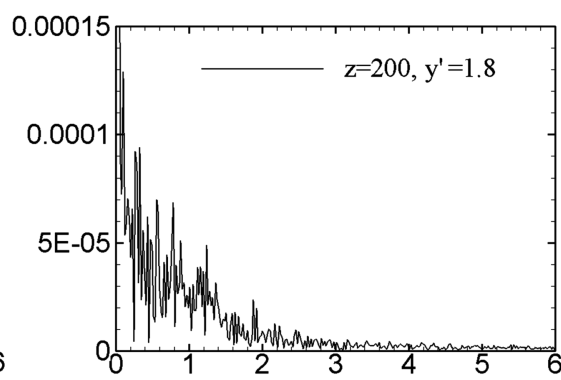

b)

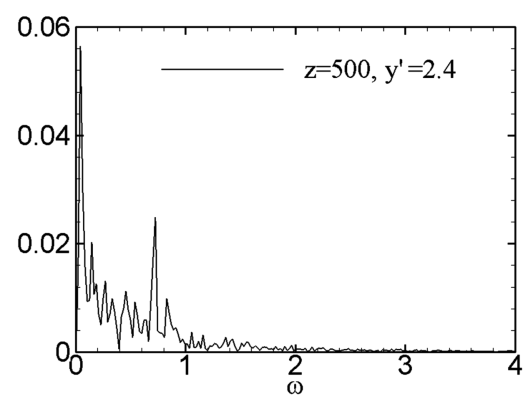

e)

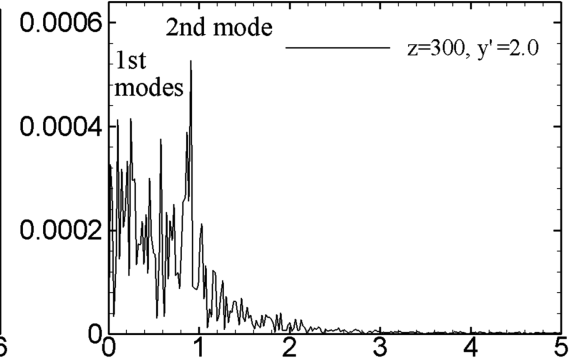

c)

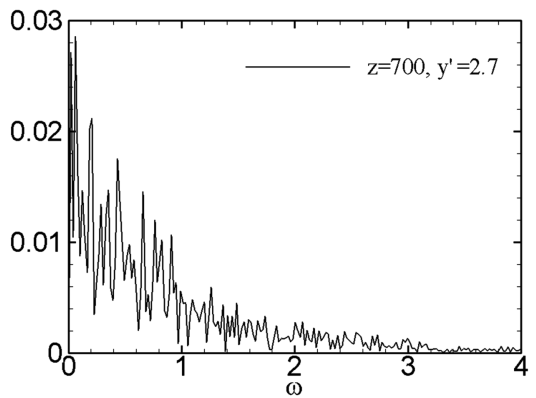

f)

Fig. 16 Frequency spectrums of streamwise velocity fluctuations $u^{\prime}$ in the leeward section.

the range of $2.1<\omega<2.3$ that is in the frequency range of the unstable second mode. The fact that the first mode is still dominating at $z=400$ (Fig. 21d) is different from the development of disturbance waves in the leeward section. At the location of $z=500$ (Fig. 21e), the amplitude of frequency in the first modes' unstablefrequency range grows slightly to $\varepsilon \approx 5 \times 10^{-4}$, whereas the amplitude of the second modes' unstable-frequency range grows rapidly to $\varepsilon=1.6 \times 10^{-4}$. At the location of $z=600$ (Fig. 21f), the second mode is stronger than the first mode, and at $\bar{z}=700$ (Fig. 21g), the second mode is dominating. There are some highfrequency peaks at $z=700$, and these peaks are the secondharmonic and third-harmonic waves of the dominating disturbance 


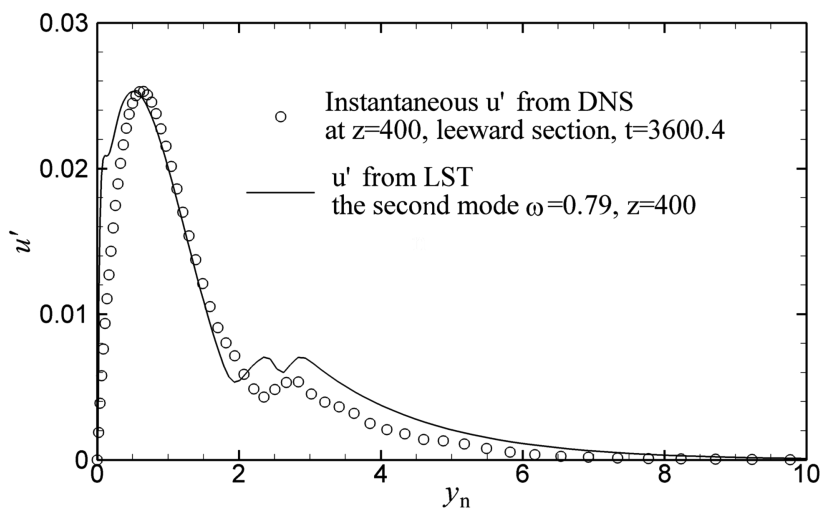

Fig. 17 Comparison of velocity fluctuation $u^{\prime}$ between LST and DNS.

wave, because the disturbance wave here is strong enough to show nonlinear effects. At $z=750$ (Fig. 21h), the spectrum shows very strong low-frequency peaks, which indicates the effects of the nonlinear mechanism. At $z=900$ (Fig. 21i), the figure shows a wideband frequency spectrum, which indicates the transition.

Figure 22 shows the frequency spectrums of wall-pressure fluctuation $\bar{p}_{w}^{\prime}$ at different six points in the wall of the windward section. Because the second mode has a strong pressure fluctuation component but the first mode's pressure fluctuation component is very weak, the pressure fluctuation is mainly affected by the second mode. This figure shows that there is a wide-spectrum bandwidth at initial location $z=100.1$, and the main second-mode disturbance wave is shown clearly at $z=300$ and becomes dominating at $z=400$, which is much later than that in the leeward section.

Figure 23 shows the neutral stability curve obtained from LST with the basic solution of the laminar blunt-cone solution and the grow routes of disturbance waves in windward section. The dashed line shows the grow route of disturbance waves. Figure 24 shows the amplitude distributions of wall-pressure perturbations of different frequencies along the cone surface in the windward section. This figure shows an exponential growth of the dominate disturbance wave $(\omega=1.6)$ in the range of $400<z<700$, with an approximately constant growth rate. From $z=400$ to 700 , the amplitude of the dominating disturbance wave is magnified by about 7000 times, with a growth rate $\bar{\alpha}=\ln 7000 /(700-400) \approx 0.030$. After

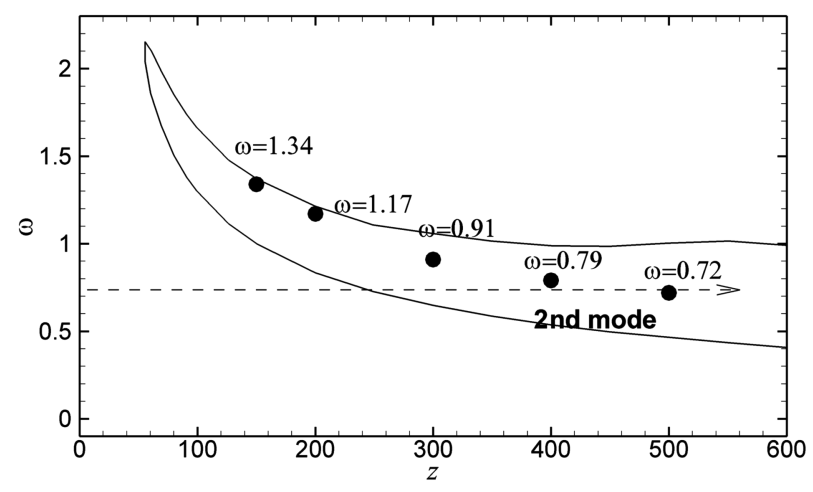

Fig. 19 Neutral stability curve of the second mode and schematic of grow routes of disturbance waves in the leeward section.

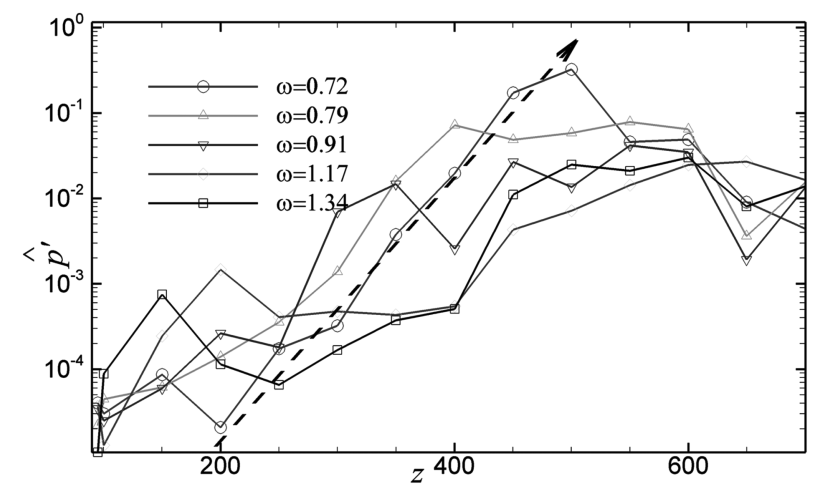

Fig. 20 Amplitude distributions of wall-pressure perturbations of difference frequencies along the cone surface in the leeward section.

comparing the growth rate of the dominating waves in the windward section and that in the leeward section (Fig. 20), we find that the difference of the growth start location is very obvious, although the difference of growth rate is not obvious. The growth start location of dominating waves in the windward section (begins at $z=400$ ) is much later than that in the leeward section (begins at $z=200$ ), which answers the question why transition is much later in the windward section.

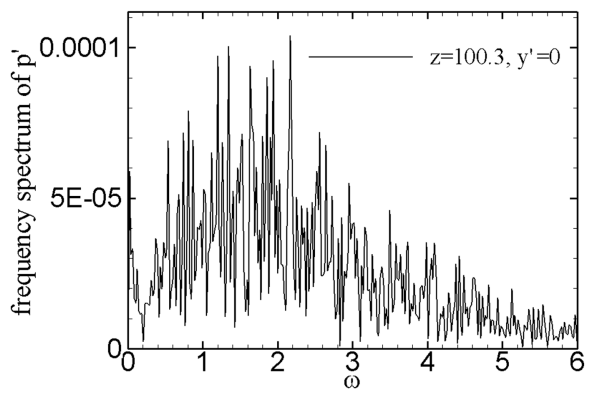

a)

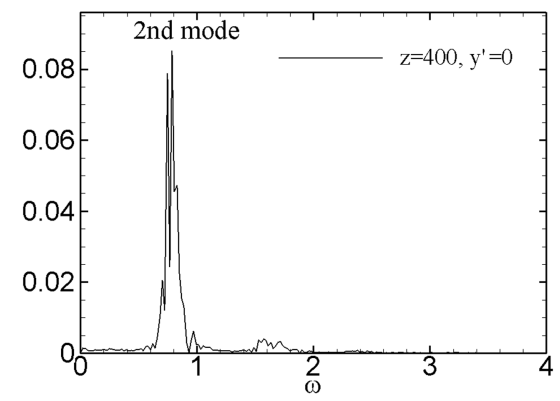

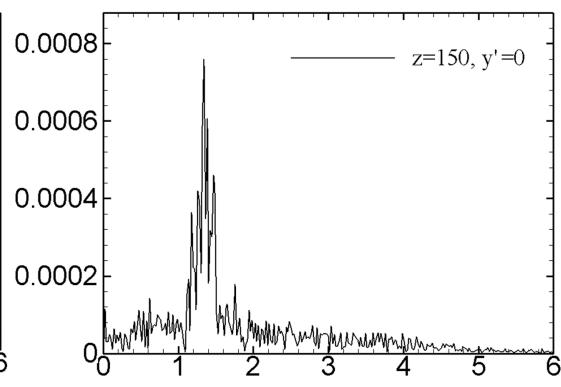

b)

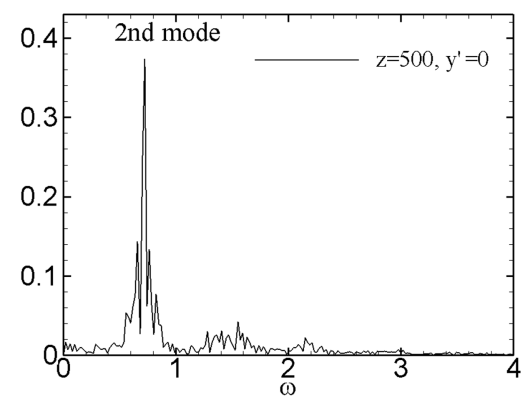

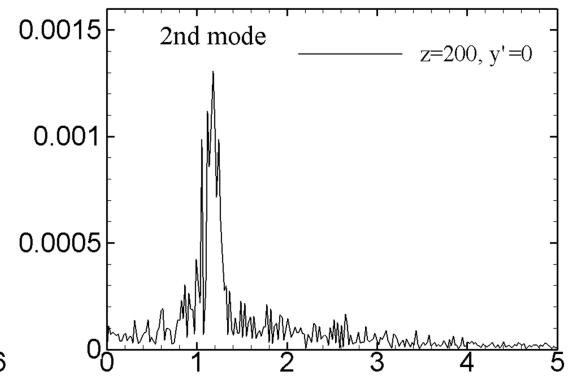

c)

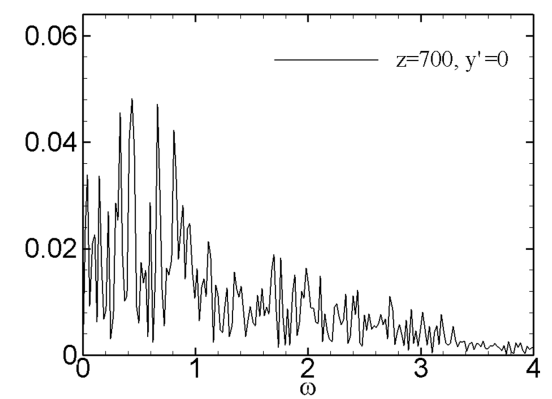

f)

Fig. 18 Frequency spectrums of wall-pressure fluctuations $p_{w}^{\prime}$ in the leeward section. 

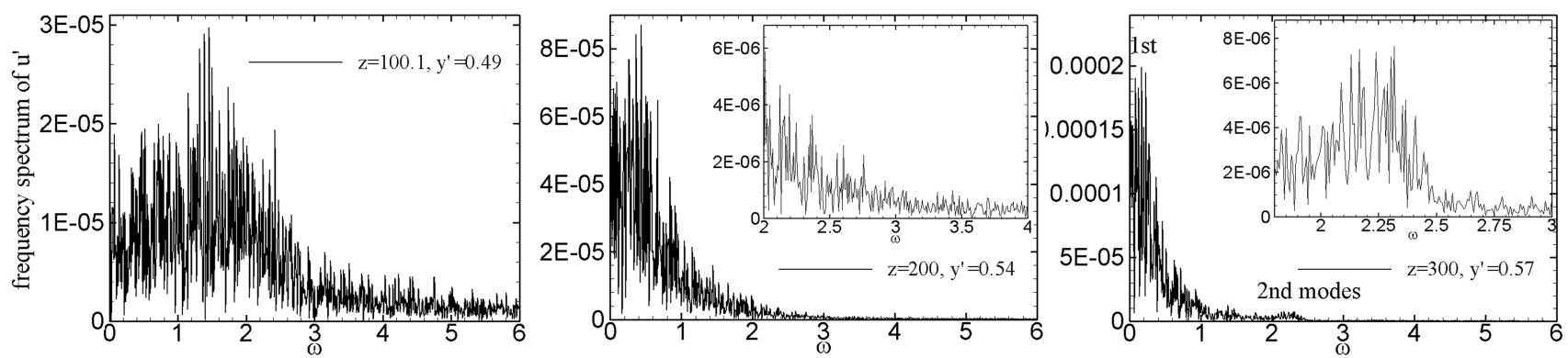

a)

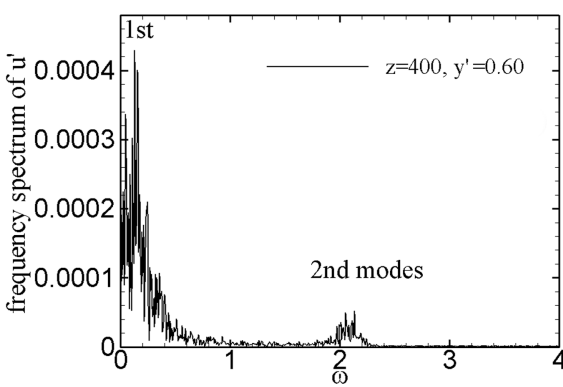

d)

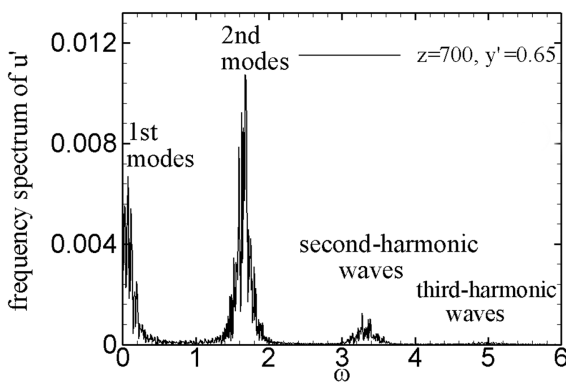

b)

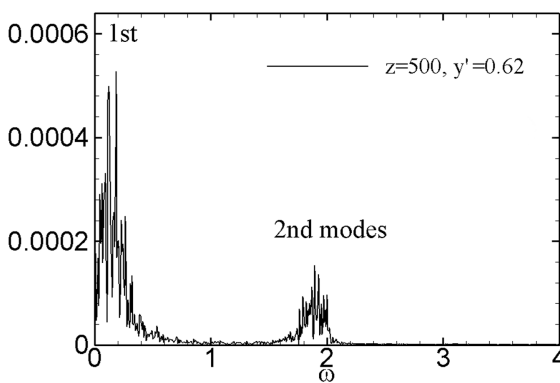

e)

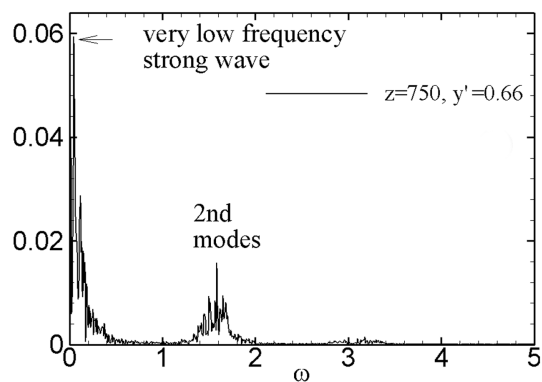

c)

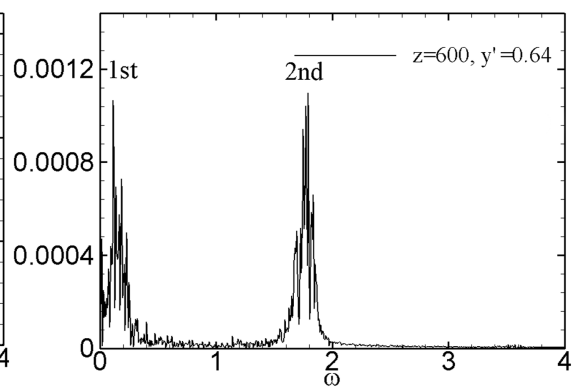

f)

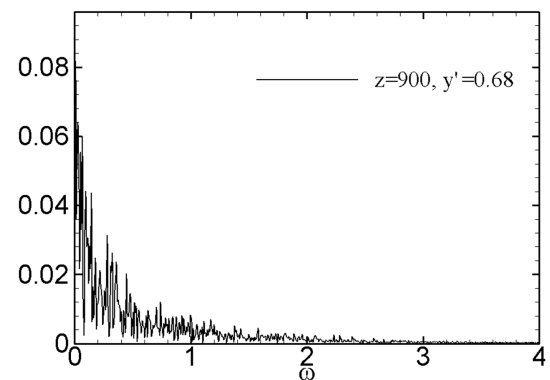

h)

i)

Fig. 21 Frequency spectrums of streamwise velocity fluctuations $u^{\prime}$ in the windward section.

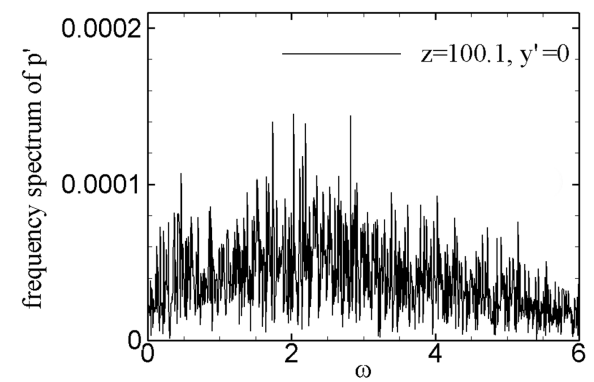

a)

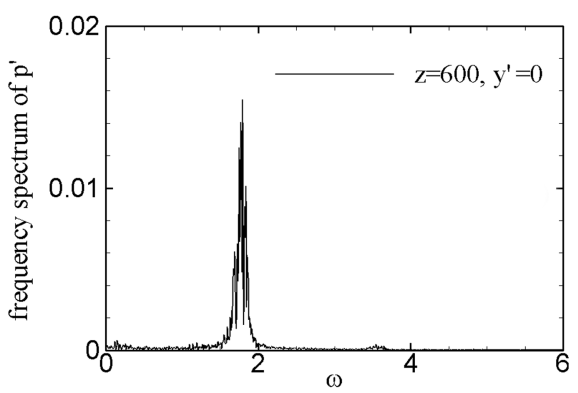

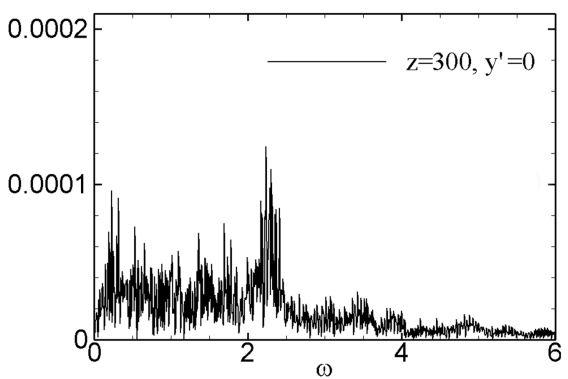

b)

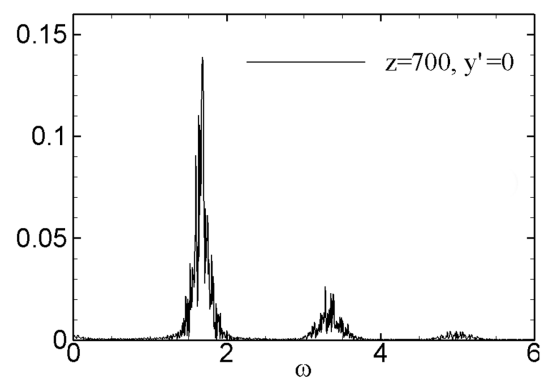

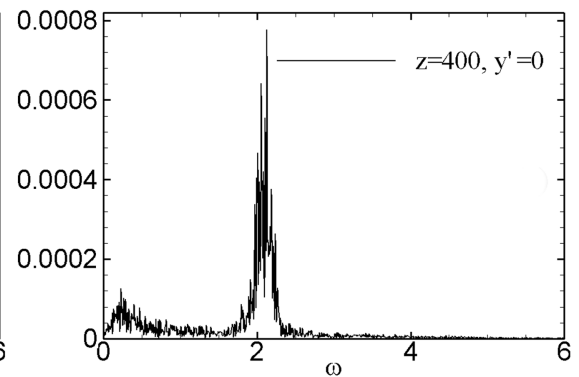

c)

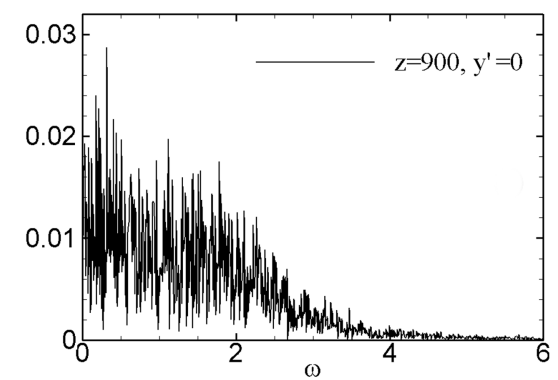

Fig. 22 Frequency spectrums of wall-pressure fluctuations $p_{w}^{\prime}$ in the windward section. 


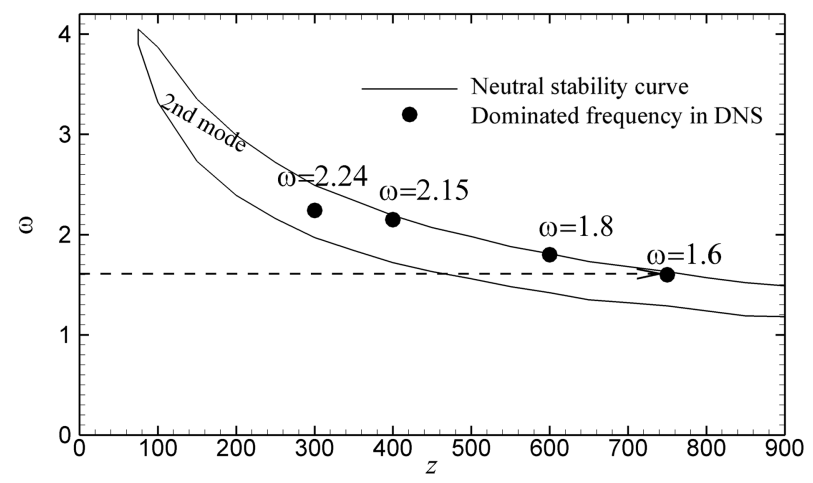

Fig. 23 Neutral stability curve of the second mode and schematic of grow routes of disturbance waves in the windward section.

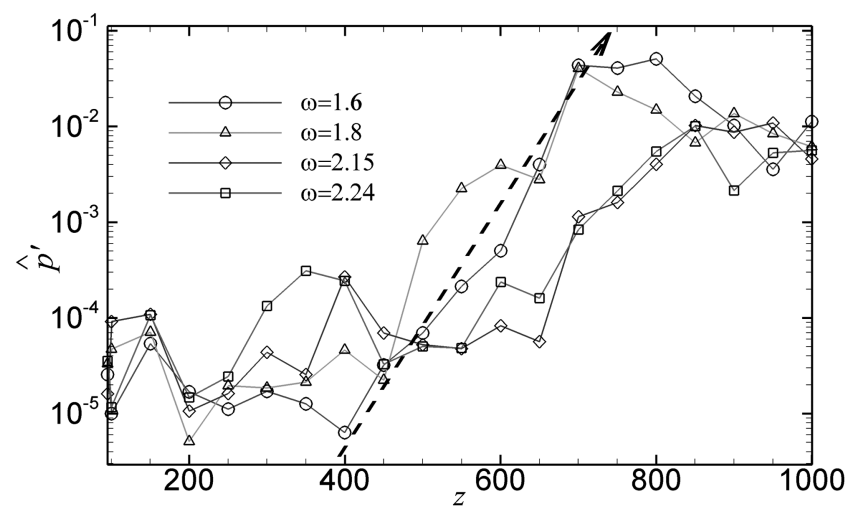

Fig. 24 Amplitude distributions of wall-pressure perturbations of difference frequencies along the cone surface in the windward section.

\section{Transition Triggered by Weak Multifrequency Disturbance}

To test the dominating waves in the transition process, additional DNS cases with multifrequency blow-and-suction disturbance are conducted. The flow and computational parameters are the same as the DNS in Sec. VI and only perturbation is different. In the DNS of this section, multifrequency blow-and-suction perturbation is imposed at the interval between $z=90$ and 100 . The perturbation contains the waves with five frequencies: $\omega=0.1,0.2,0.3,0.4$, and 0.5 . The amplitude of each wave is 1.25 times that for the adjacent higher-frequency wave, and total amplitude is $A=0.01$. These frequencies are in the unstable range of the first mode.

Figures $25 \mathrm{a}$ and $25 \mathrm{~b}$ show the friction coefficient in the leeward and windward sections of this DNS. Figure 25a shows that the transition onset is located at $z=500$ and the transition peak is located at $z=630$ in the leeward section, and Fig. 25b shows that transition onset is located at $z=750$ and the transition peak is located at $z=960$ in the windward section. Transition locations in these multifrequency-disturbance cases agree well with those in the cases of random disturbance.

Figure 26 shows the frequency spectrum of streamwise velocity fluctuations at the three different points at streamwise locations that are 0.6-0.7 times the boundary layer's thickness in the leeward section. At $z=100.3$, just behind the disturbance range, there are five dominating frequencies: $\omega=0.1,0.2,0.3,0.4$, and 0.5 . These frequencies are the same as the initially imposed perturbation's frequencies, with an amplitude of about $10^{-4}$. These waves are all in the frequency range of the unstable first mode. The amplitude in the high-frequency range is at least one order smaller than that of the five main frequency peaks. Figure 26 shows that the amplitude peak in the unstable second mode's frequency range is significant at $z=200$ and becomes dominating at $z=400$. This result shows that even in the case in which the initial disturbance of first mode is much stronger than the second mode, the second mode can still become dominating at the downstream location, because the second mode's growth rate is much higher than the first mode's.
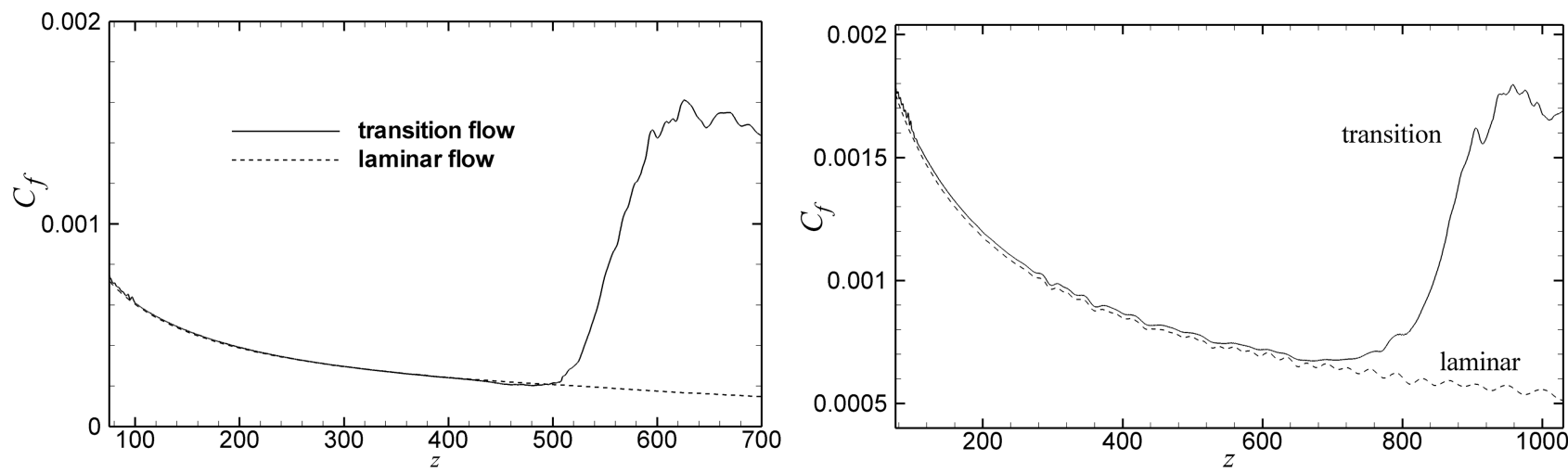

a)

b)

Fig. 25 Distribution of $C_{f}$ in the leeward (left) and windward section (right).
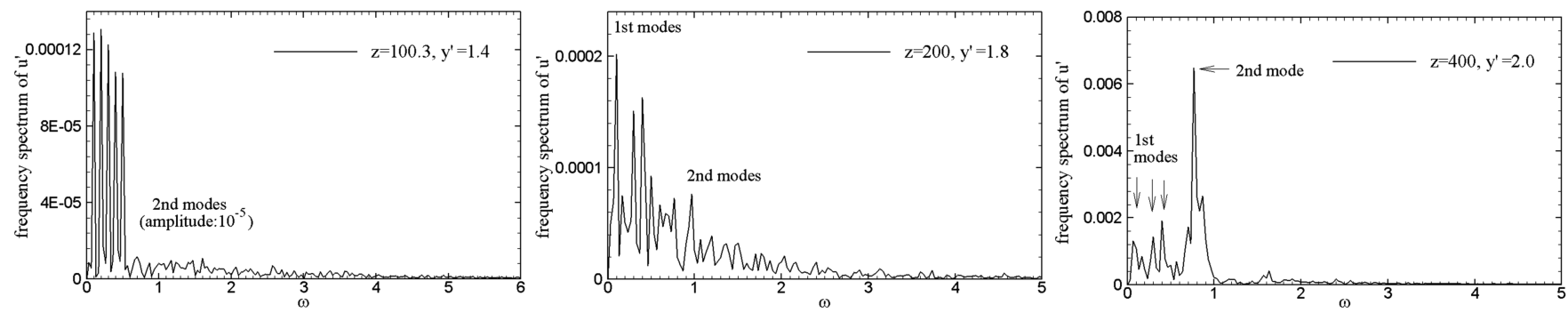

Fig. 26 Frequency spectrum of streamwise velocity fluctuation $u^{\prime}$ in the boundary layer of the leeward section. 


\section{Conclusions}

Direct numerical simulation of transition flow around a blunt cone with freestream Mach number $M_{\infty}=6$, nose radius Reynolds number $R e=10,000$, and $\mathrm{AOA}=1 \mathrm{deg}$ is conducted using a seventh-order WENO scheme with an eighth-order central finite difference scheme. The wall blow-and-suction disturbances (including random perturbation and multifrequency perturbation with maximum amplitude of disturbance velocity of $1 \%$ of freestream velocity) are used to trigger the transition. Transition locations for random-perturbation cases and multifrequencyperturbation cases agree well with each other. The transition onset is located at about 500 times the nose radius in the leeward section (leeward meridian plane) and 750 times the nose radius in the windward section (windward meridian plane).

The second-mode waves are deemed to be the dominating disturbance that triggers the transition. It is found that the growth of disturbance waves in the windward section starts much later than that in the leeward section. Therefore, transition is more delayed in the windward section than in the leeward section.

\section{Acknowledgments}

This work was supported by the National Natural Science Foundation of China (grants 10632050 and 10502052) and Knowledge Innovation Projects of the Chinese Academy of Sciences. The authors would like to thank the Supercomputing Center of the Chinese Academy of Sciences (SCCAS) and the Shanghai Supercomputer Center (SSC) for providing computer time. They also thank Zhou Heng, Luo Jiseng, and Cao Wei of Tianjing University for the code SAYR of linear stability theory analysis and for their helpful comments.

\section{References}

[1] Schneider, S. P., "Flight Data for Boundary Layer Transition at Hypersonic and Supersonic Speeds," Journal of Spacecraft and Rockets, Vol. 36, No. 1, 1999, pp. 8-20.

[2] Horvath, T. J., Berry, S. A., Hollis, B. R., Chang, C. L., and Singer, B. A., "Boundary Layer Transition on Slender Cones in Conventional and Low Disturbance Mach 6 Wind Tunnels," AIAA Paper AIAA-20022743, 2002.

[3] Mack, L. M., "Boundary Layer Linear Stability Theory," AGARD, Rept. 709, Neuilly-sur-Seine, France, 1984.

[4] Stetson, K. F., and Kimmel, R. L., "On Hypersonic Boundary Layer Stability," AIAA Paper 92-0737, 1992.

[5] Stetson, K. F., and Kimmel, R. L., "On the Breakdown of a Hypersonic Laminar Boundary Layer," AIAA Paper 93-0896, 1993.

[6] Maslov, A. A., Mironov, S. G., and Shiplyuk, A. A., "Hypersonic Flow Stability Experiments," AIAA Paper 2002-0153, 2002.

[7] Zhong, X., and Ma, Y., "Boundary-Layer Receptivity of Mach 7.99 Flow over a Blunt Cone to Freestream Acoustic Waves," Journal of Fluid Mechanics, Vol. 556, June 2006, pp. 55-103. doi:10.1017/S0022112006009293

[8] Saric, W. S., Reed, H. L., and Kerschen, E. J., "Boundary-Layer Receptivity to Freestream Disturbances," Annual Review of Fluid Mechanics, Vol. 34, 2002, pp. 291-319.

doi:10.1146/annurev.fluid.34.082701.161921

[9] Ma, Y., and Zhong, X., "Receptivity of a Supersonic Boundary Layer over a Flat Plate, Part 1: Wave Structures and Interactions," Journal of Fluid Mechanics, Vol. 488, July 2003, pp. 31-78. doi: $10.1017 / \mathrm{S} 0022112003004786$

[10] Ma, Y., and Zhong, X., "Receptivity of a Supersonic Boundary Layer over a Flat Plate, Part 2: Receptivity to Freestream Sound," Journal of Fluid Mechanics, Vol. 488, July 2003, pp. 79-121. doi: $10.1017 / \mathrm{S} 0022112003004798$

[11] Zhong, X., "Leading-Edge Receptivity to Freestream Disturbance Waves for Hypersonic Flow over a Parabola," Journal of Fluid Mechanics, Vol. 441, Aug. 2001, pp. 315-367. doi:10.1017/S0022112001004918

[12] Tumin, A., "Multimode Decomposition of Spatially Growing Perturbations in a Two-Dimensional Boundary Layer," Physics of Fluids, Vol. 15, July 2003, Paper 2525. doi:10.1063/1.1597453

[13] Tumin, A., "Three-Dimensional Spatial Normal Modes in Compressible Boundary Layers," Journal of Fluid Mechanics, Vol. 586,
Sept. 2007, pp. 295-322.

doi: $10.1017 /$ S002211200700691X

[14] Tumin, A., Wang, X., and Zhong, X., "Direct Numerical Simulation and the Theory of Receptivity in a Hypersonic Boundary Layer," Physics of Fluids, Vol. 19, No. 1, 2007, Paper 014101. doi:10.1063/1.2409731

[15] Su, C., and Zhou, H., "Stability Analysis and Transition Prediction of Hypersonic Boundary Layer over a Blunt Cone with Small Nose Bluntness at Zero Angle of Attack," Applied Mathematics and Mechanics (English Edition), Vol. 28, No. 5, 2007, pp. 563-572. doi:10.1007/s10483-007-0501-1

[16] Moin, P., and Mahes, K., "Direct Numerical Simulation: A Tool in Turbulence Research," Annual Review of Fluid Mechanics, Vol. 30, 1998, pp. 539-578. doi:10.1146/annurev.fluid.30.1.539

[17] Guarini, S. E., Moser, R. D., Shariff, K., and Wray, A., "Direct Numerical Simulation of a Supersonic Turbulent Boundary Layer at Mach 2.5," Journal of Fluid Mechanics, Vol. 414, July 2000, pp. 1-33. doi: $10.1017 / \mathrm{S} 0022112000008466$

[18] Maeder, T., Adams, N. A., and Kleiser, L., "Direct Simulation of Turbulent Supersonic Boundary Layer by an Extended Temporal Approach," Journal of Fluid Mechanics, Vol. 429, Feb. 2001, pp. 187 216. doi:10.1017/S0022112000002718

[19] Rai, M. M., Gatski, T. B., and Erlebacher, G., "Direct Numerical Simulation of Spatially Evolving Compressible Turbulent Boundary Layers," AIAA Paper 95-0583, 1995.

[20] Pirozzoli, S., and Grasso, F., "Direct Numerical Simulation and Analysis of a Spatially Evolving Supersonic Turbulent Boundary Layer at $M=2.25$," Physics of Fluids, Vol. 16, No. 3, 2004, pp. 530-545. doi:10.1063/1.1637604

[21] Li, X. L., Fu, D. X., and Ma, Y. W., "DNS of Compressible Turbulent Boundary Layer over a Blunt Wedge," Science in China Series G, Vol. 48, No. 2, 2005, pp. 129-141. doi:10.1360/03yw0300

[22] Li, X. L., Fu, D. X., and Ma, Y. W., "Direct Numerical Simulation of a Spatially Evolving Supersonic Turbulent Boundary Layer at $M a=6$," Chinese Physics Letters, Vol. 23, No. 6, 2006, pp. 1519-1522. doi:10.1088/0256-307X/23/6/045

[23] Gao, H., Fu, D. X., Ma, Y. W., and Li, X. L., "Direct Numerical Simulation of Supersonic Turbulent Boundary Layer," Chinese Physics Letters, Vol. 22, No. 7, July 2005, pp. 1709-1712. doi:10.1088/0256-307X/22/7/041

[24] Zhou, Y., Li, X. L., Fu, D. X., and Ma, Y. W., "Coherent Structures in Transition of a Flat-Plate Boundary Layer at $M a=0.7$," Chinese Physics Letters, Vol. 24, No. 1, 2007, pp. 147-150. doi: 10.1088/0256-307X/24/1/040

[25] Jiang, G. S., and Shu, C. W., "Efficient Implementation of Weighted ENO Schemes," Journal of Computational Physics, Vol. 126, No. 1, June 1996, pp. 202-228. doi: $10.1006 /$ jcph. 1996.0130

[26] Ren, Y. X., Liu, M., and Zhang, H., "A Characteristic-Wise CompactWENO Scheme for Solving Hyperbolic Conservation Laws," Journal of Computational Physics, Vol. 192, No. 2, Dec. 2003, pp. 365386. doi:10.1016/j.jcp.2003.07.006

[27] Wang, Z. J., and Chen, R. F., "Optimized Weighted Essentially Nonoscillatory Schemes for Linear Waves with Discontinuity," Journal of Computational Physics, Vol. 174, No. 1, Nov. 2001, pp. 381-404. doi:10.1006/jcph.2001.6918

[28] Martin, M. P., Taylor, E. M., Wu, M., and Weirs, V. G., "A BandwidthOptimized WENO Scheme for the Effective Direct Numerical Simulation of Compressible Turbulence," Journal of Computational Physics, Vol. 220, No. 1, Dec. 2006, pp. 270-289. doi:10.1016/j.jcp.2006.05.009

[29] Borges, R., Carmona, M., Costa, B., Don, W. S., "An Improved Weighted Essentially Nonoscillatory Scheme," Journal of Computational Physics, Vol. 227, No. 6, Mar. 2008, pp. 3191-3211. doi:10.1016/j.jcp.2007.11.038

[30] Stetson, K. F., "Hypersonic Boundary Layer Transition Experiments," U.S. Air Force Wright Aeronautical Labs., TR-80-3062, WrightPatterson AFB, OH, 1980.

[31] Fu, D. X., Ma, Y. W., and Zhang, L. B., "Direct Numerical Simulation of Transition and Turbulence in Compressible Mixing Layer," Science in China, Series A (Mathematics, Physics, Astronomy and Technological Sciences), Vol. 43, No. 4, 2000, pp. 421-429.

[32] Adams, N. A., "Direct Simulation of the Turbulent Boundary Layer Along a Compression Ramp at $M=3$ and $R e=1685$," Journal of 
Fluid Mechanics, Vol. 420, Oct. 2000, p. 47. doi: $10.1017 / \mathrm{S} 0022112000001257$

[33] Egorov, I. V., Fedorov, A. V., and. Soudakov, V. G., "Direct Numerical Simulation of Disturbances Generated by Periodic Suction Blowing in a Hypersonic Boundary Layer," Theoretical and Computational Fluid Dynamics, Vol. 20, No. 1, 2006, pp. 41-54. doi:10.1007/s00162-005-0001-y

[34] Zhang, Y. D., Fu, D. X., Ma, Y. W., and Li, X. L., "A High-Order Unsteady Shock-Fitting Scheme," Chinese Journal of Computational Physics, Vol. 24, No. 5, 2007, pp. 536-541 (in Chinese).

[35] Zhong, X., "High-Order Finite-Difference Schemes for Numerical Simulation of Hypersonic Boundary-Layer Transition," Journal of Computational Physics, Vol. 144, No. 2, Aug. 1998, pp. 662-709. doi:10.1006/jeph.1998.6010

[36] Stetson, K. F., Thompson, E. R., Donaldson, J. C., and Siler, L. G., "Laminar Boundary Layer Stability Experiments on a Cone at Mach 8,
Part 2: Blunt Cone," AIAA Paper 84-0006, 1984.

[37] Herbert, T. H., and Esfahanian, V., "Stability of Hypersonic Flow over a Blunt Body," AGARD CP 514, 28-1-28-12, 1993.

[38] Fiala, A., Hillier, R., Mallinson, S. G., and Wijestinghe, H. S., "Heat Transfer Measurement of Turbulent Spots in a Hypersonic Blunt-Body Boundary Layer," Journal of Fluid Mechanics, Vol. 555, May 2006, pp. 81-111.

doi:10.1017/S0022112006009396

[39] Krishnan, L., and Sandham, N. D., "Effect of Mach Number on the Structure of Turbulent Spots," Journal of Fluid Mechanics, Vol. 566, Nov. 2006, pp. 225-234.

doi:10.1017/S0022112006002412 